\title{
Topological approach for assessment of electromagnetic interferences to support mechatronic conceptual design
}

\author{
Mouna Kharrat ${ }^{1,2, *}$, Olivia Penas ${ }^{1}$, Nourhene Abdeljabbar Kharrat ${ }^{1,2}$, Régis Plateaux ${ }^{1}$, Jean-Yves Choley $^{1}$, \\ Jamel Louati ${ }^{2}$, Hassen Trabelsi ${ }^{2}$, and Mohamed Haddar ${ }^{2}$ \\ ${ }^{1}$ QUARTZ Laboratory (EA7393), Institut Supérieur de Mécanique de Paris (SUPMECA), 3, rue Fernand Hainaut, \\ 93407 Saint-Ouen Cedex, France \\ ${ }^{2}$ Laboratory Mechanical, Modeling and Manufacturing, National Engineering School of Sfax (ENIS), BP 1173-3038-Sfax, Tunisia
}

Received: 9 April 2019 / Accepted: 1 October 2020

\begin{abstract}
The conceptual design is a crucial phase for the System Architects to evaluate 3D architecture concepts of mechatronic systems mainly with regard to the ElectroMagnetic Compatibility (EMC). Our research work deals with the combination of electromagnetic modeling and a topological approach to support the qualitative and quantitative ElectroMagnetic Interferences (EMI) evaluation process within the MBSE SAMOS approach as of the conceptual design. For a given interference, the analysis of topological models allows the qualitative identification of the existence of victims with their associated potential aggressors, based on the electrical schema of interacting components. Then, once the potential EMIs have been qualitatively identified, a quantitative evaluation can be performed based on the predefined electromagnetic and geometrical requirements, and on the analysis of the identified physical coupling law. Finally, this approach has been applied to the alien crosstalk occurring in an electrical vehicle powertrain, with a quantitative evaluation based on the two following methods: an analytical approach and Kron's approach.
\end{abstract}

Keywords: Mechatronic design / architecture evaluation / topological modeling / electromagnetic interferences identification / conceptual design

\section{Introduction}

\subsection{EMC issues in mechatronic systems}

The high integration of Information Technology (IT) based Electronic and Electric (E/E) devices in common systems makes them increasingly mechatronic. Notably in the automotive and aeronautical sectors where the context of transport electrification encourages system architects to define new innovative architecture during the conceptual design phase; the development of such forerunner concepts can generate considerable risks and costs during the further design stages [1]. Indeed, the physical integration of an ever-increasing number of multi-domain components in a reduced volume can create unwanted multi-physical couplings between them.

Then, even if the performance of such systems has to be evaluated under these multi-physical constraints, the corresponding simulations are typically performed once the concept's physical architecture has been selected, i.e. in the detailed design phase. This late multi-physical

\footnotetext{
* e-mail: mouna.kharrat53@gmail.com
}

evaluation results in arduous tradeoffs on the final 3D architecture, usually based on many iterations between multiple experts of different disciplines. Indeed at this design stage, the possibilities of compromise are often limited to only a few positional adjustments or some costly protective solutions to limit unwanted physical disturbances, and experts do not have the possibility to fully challenge the proposed 3D architecture. In this context, as most product development costs are generally incurred at the end of the design phase, late changes to the architecture at the detailed design phase can result in a negative effect on product quality, cost and performance. As a result, some authors propose an integrated design approach for mechatronic systems to support designers during the conceptual design phase $[2,3]$. Then, the $3 \mathrm{D}$ architecture evaluation under the multi-physical constraints of such products, as of the conceptual design, becomes a crucial step requiring a multidisciplinary approach based on System Engineering (SE) in order to cope with all the previous considerations.

Electro-Magnetic Compatibility (EMC) issues have become critical to System architects due to the increased use of E/E systems in advanced technologies. Notably in 
the automotive and aeronautic sectors, numerous current systems epitomize such an evolution: autonomous systems, advanced driver-assistance systems, ubiquitous IoT systems, customized comfort. Indeed, it typically represents more than $30 \%$ of the cost of a typical modern car [4]. This ongoing demand for even more electronic systems results today in an increasing number of EMC problems [5,6], which designers are finding difficult to solve without increasing costs and safety risks [7,8]. In this respect, Zhao et al. provide an insight into some current research on EMC questions and solutions deployed, particularly in vehicle $\mathrm{E} / \mathrm{E}$ devices [9].

Thus, the design of such systems requires the consideration of EMC constraints. It should be assessed as soon as possible in order to limit the risk of ElectroMagnetic Interferences (EMI) in response to industrial technological challenges and existing legislative requirements and standards [5]. Unfortunately, electromagnetic characteristics are often only specified once the product is manufactured according to the customer's specifications [10], and the implementation of many EMC standards is difficult in the early design phases when the knowledge of components may still be uncertain.

Additionally, manufacturers may experience emergent EMI phenomena during the integration phase of the complete system [4], even if each component has successfully undergone experimental tests in compliance with EMC standards. Accordingly, Burnham highlighted this problem for vehicle integrators and suggested that EMC issues should be taken into account both at the early design stage and at the vehicle level [11].

Finally, designing mechatronic systems that ensure the integration of their (E/E) components whilst both satisfying EMC, and not increasing costs and safety risks, is a major current industrial issue [8].

In summary, the increasing use of $\mathrm{E} / \mathrm{E}$ systems on the one hand, and the lack of flexibility in the placement and selection of electronic modules on the other hand, make EMC problems increasingly critical for mechatronics system designers. Additionally, it is also necessary to have an EMC analysis at the system level from the early stages of the vehicle design process [4], in order to be able to assess the EMI of the various 3D architecture alternatives.

Therefore, to tackle these challenges, our research work deals with the identification of the potential EMI generated between interacting components, based on a topological analysis and the $3 \mathrm{D}$ positioning of $\mathrm{E} / \mathrm{E}$ components.

\subsection{Research background}

\subsubsection{Integration in the $\mathrm{V}$-cycle}

There are two main branches of the V-design cycle. The first branch consists of the product development process. This branch is generally divided into three main phases: the pre-design phase, the preliminary design phase and the detailed design phase. In the pre-design phase, the first step is the analysis of the customer's needs, and the generation of requirements. Based on these derived requirements, the System Architect provides a functional and then logical architecture. Then, the System Architect proposes alternative physical architectures (with components) to be evaluated. Once the concept architecture is selected, the preliminary and detailed design is carried out. Moreover, EMC performance specifications (in immunity and emission) intervene at different levels of the $\mathrm{V}$ cycle in order to be able to assess the aggression levels and to justify an architecture choice that meets these EM constraints. For example, at the component level: the system architect may reject $\mathrm{E} / \mathrm{E}$ components identified as sensitive for the mission; at the equipment (subsystem) level: the system architect may develop EMC performance specifications for equipment (e.g. electronic enclosures and cabling) but these do not necessarily prove that the equipment will satisfy the system requirements; at the system level: the system architect has to verify that the system fulfils the normative constraints. Finally, the verification of the derived requirements is carried out by the system architect through detailed geometrical and multi-physical (EM) simulations. This last step consists of the second ascending branch of the $\mathrm{V}$ cycle. In general, it consists of validating the technical choices made in order to confirm the manufactured system through the qualification of the subsystems and the final certification of the complete system. In this context, we propose to integrate a preliminary evaluation of the 3D architecture under physical constraints for the choice of the concept (between logical and physical architecture) in order to select an architecture that meets the $3 \mathrm{D}$ and physical constraints, long before the detailed design phase (Fig. 1).

\subsubsection{SAMOS approach and 3D multi-physical sketcher}

Designing systems which integrate multi-domain components whose proximity often leads to multi-physical interactions, is today a major challenge. Indeed, as multiphysical phenomena are highly dependent on the physical 3D architecture, and as design costs are mainly committed in the first design phases $[12,13]$, it is necessary to be able to evaluate 3D architecture alternatives under multi-physical constraints from the conceptual design phase. In parallel, Model-Based Systems Engineering (MBSE) efficiently supports the multidisciplinary approach required by the design of such systems [14-16]. Thus, our research work focuses on the extension of the SAMOS (Spatial Architecture based on Multi-physics and Organization of Systems) MBSE approach [17], developed by R. Barbedienne for thermal constraints [18], to the EM constraints [15]. SAMOS supports the evaluation of 3D physical architecture under multi-physical constraints during the conceptual design phase. The implemented SAMOS-based 3D multiphysical sketcher improves the design's data consistency and traceability through Modelto-Model transformations, while ensuring, at early design stages, a collaborative framework between System architects, 3D architects and multidisciplinary experts. The current work described in this paper focuses on the identification of EMI risks between the 3D architecture components through a topological approach, and its integration into the SAMOS framework. 


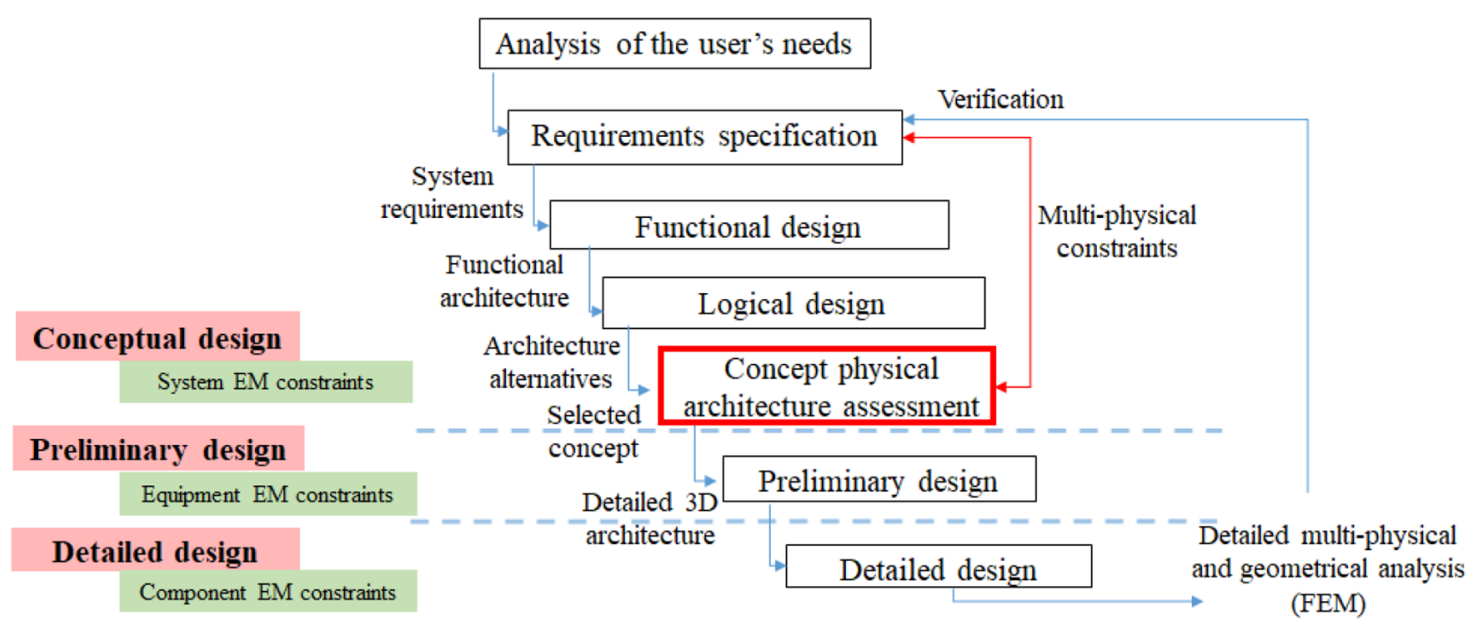

Fig. 1. Integration in the first branch of the V-model.

\subsubsection{Topological approach for multi-physics}

In parallel, the main objective of our topology related work is to develop a single optimal modeling of any complex mechatronic system. It addresses the connectivity study of behavioral models in a multi-level and multi-scale approach, in order to highlight continuity and connectivity in the design phase of such systems. To achieve this purpose, significant research has already been carried out through the studies of Plateaux [19], Chaabane [20] and Abdeljabbar Kharrat [21]. In this work, topological collections support the system topology, i.e. the connection between the different components of a mechatronic system and the transformations that are used to specify the local behavior laws of each of these components.

Plateaux has used topological modeling both to guarantee model consistency and data continuity during the mechatronic design phase $[22,23]$ and to formalize multiphysics modeling of mechatronic systems, whilst retaining the link between functional requirements and feasible architectures [19].

In her research work, Chaabane has developed a topological approach for modelling mechatronic systems, with a unified representation using the MGS language [24]. This approach makes it possible to model a dynamic system and to specify the local behavioral laws of each component of the system by dissociating its topology from its physics [20]. However, this topological approach must be evaluated with a more complex system that integrates both topology and multi-physical constraints within the same model.

As a result, Abdeljabbar Kharrat has developed a new topological methodology for modelling multi-physical systems, which has been implemented using dynamic models with a variable topology. The integration of topological modification has been applied to a pogo-stick [21] and validated with the modeling of multi-physical systems, by studying the case of piezoelectric structures (piezoelectric stack and lattice piezoelectric) [25].

\subsection{Research objective}

The intent of this paper lies in combining electromagnetic modeling and a topological approach to support an EMI evaluation process during the conceptual design of mechatronic systems, in order to reduce the risk of late and costly architecture changes.

The paper is organized as follows. Section 2 describes the state-of-the-art of EMI risk evaluation and its related existing topological approaches. Then, Section 3 presents the electromagnetic modeling using this topological approach for EMI evaluation, detailing its integration into the SAMOS framework, before focusing on the alien crosstalk identification process, and also addresses the EMI quantitative evaluation. Section 4 describes its application in an Electric Vehicle Power Train (EVPT) scenario. Section 5 proposes some discussions and future work. Finally, Section 6 summarizes our proposal and concludes the paper.

\section{State of the art}

\subsection{EMI risk evaluation}

The increasing level of integration in multi-domain systems requires that the risk of EMI, taking into account the $3 \mathrm{D} \mathrm{E} / \mathrm{E}$ component architecture, should be tackled. The assessment of such EMI issues is typically achieved with various simulation tools, such as the Transmission Line Modelling (TLM) [26], the Method of Moment (MoM) and Boundary Element (BE) [27], the Finite Difference Time Domain (FDTD) [28] or multiconductor transmission line (MTL) [29]. However, such evaluations are usually performed late in the design process, once a numerical or physical prototype has been built, and thus they are both overly time consuming and costly in view of the increasingly tight time constraints of current product development programs. Therefore, the analysis of potential EMC issues must be addressed as 
early as possible during the design stages, before the detailed design phase where architectural changes are highly burdensome.

Concerning the EMI risk assessment, the generation of electromagnetic couplings causing EMC problems that degrade the functioning of electrical equipment or cause its failure, require three main elements: the source of interference, the coupling path and sensitive equipment. Maurice, in his research [30], proposed a disturbance risk formula $R=\frac{W S}{P_{c}}$, where $W$ is related to the energy of sources (aggressors), $S$ is the transfer function that transmits the aggressors energy to the victims and $P_{c}$ is the immunity threshold power of the victim components. W and $P_{c}$ are determined by the electrical characteristics (voltage, current, emitting spectrum density) contained in the electrical diagram. S depends on the interaction law of the identified EMI, including component geometrical information. Another approach consists of determining both the probability of disturbing a component for a given stress level (voltage, current) higher than a level of X0, and the probability that the stress signal exceeds the value of X0. The combination of these two functions (linked to some probability distribution assumptions) makes it possible to express the risk of the EMC failure and the margin relative to the operating safety objective [31].

\subsection{Electromagnetic (EM) modeling based on topology}

Regarding research on EM modeling based on topology, numerous approaches have been proposed [32-35] around the concept of "EM topology" that provides a useful methodology for the analysis of complex EM interactions [36-38]. In fact, it consists of associating intuitive interactions between the defined volumes in a junctiontube interaction on a topological network, where the junction represents a sub-volume and the tube represents an interaction path. It supports the definition of the EM interfaces before the related numerical implementation. For example, Genender et al. propose to determine the realistic risk of different electromagnetic environments, based on the combination of the descriptive "EM topology", statistical-based models and fault tree analysis [39]. "EM topology" was well suited to highly shielded systems with obvious EM boundaries, but this kind of qualitative topology cannot address the EMC study of "open" systems, as it develops a matrix equation without any differential geometry unlike the tensorial approach. In this context, Maurice has developed a mathematical technique based on the topological tensorial analysis of networks developed by Kron to compute all the interactions encountered in EMC in large systems $[30,40]$.

Finally, even if many numerical methods and topological approaches for the EMI assessment exist, few of them address the coupling of both topological modeling and the $3 \mathrm{D}$ positioning between $\mathrm{E} / \mathrm{E}$ interacting components at the early design stages to support the EMC prediction.

\section{EM modeling using a topological approach for EMI evaluation}

\subsection{Proposed approach integration in the SAMOS framework}

The SAMOS approach, developed by Barbedienne et al., is based on the transformation of bilateral models from three different environments: the SysML model environment, the 3D environment and the physical behavior modelling environment [17]. Previous works have focused mainly on the evaluation of 3D architecture of concepts under thermal constraints. For this purpose, they have developed two SysML extensions: GERTRUDE for geometry modeling (shape and position of 3D components) [41] and THERESE for thermal constraints modeling [18].

The SAMOS framework supports the evaluation of 3D architecture under multiphysical constraints, during the conceptual design phase [15]. As the 3D positioning between $\mathrm{E} / \mathrm{E}$ interacting components impacts EMC system performances, a 3D architecture has to be assessed as early as possible in order to verify that the chosen positioning of components meets the predefined EM requirements. Therefore, our work deals with the 3D architecture assessment under EM constraints. This section describes our approach to integrating the EM couplings evaluation into the SAMOS framework (Fig. 2).

This process begins with the System modeling, with the definition of EM requirements though the EMILE (ElectroMagnetic Interactions Layout Extension) SysML extension and geometrical requirements provided by the GERTRUDe profile [41]. These requirements relate to the components' properties: EM emission/ immunity levels, the applicable standards to be met by the system, physical parameters, environmental solicitations and geometrical information (known or specified shapes, dimensions, positioning constraints, etc.). This System modeling is also comprised of electromagnetic modeling, including known associated EM couplings.

Therefore, to evaluate the EMI disturbances, we propose to first identify the EM couplings through a topological approach, in order to later quantitatively evaluate them in a given 3D architecture. This EMI disturbances evaluation is based on the physical and topological modeling of the system's E/E structure (be it equipment, cable or antenna). The topological analysis allows for the identification of all the structurally possible EM disturbances, that should then be quantitatively evaluated through mathematical models or numerical simulation modeling, based on the 3D geometrical information (components geometry and positioning) provided by the 3D architecture.

In the next section, we detail the topological approach developed for the identification of the alien crosstalk, as this EM coupling greatly depends on the cables' geometry and their relative positioning. 


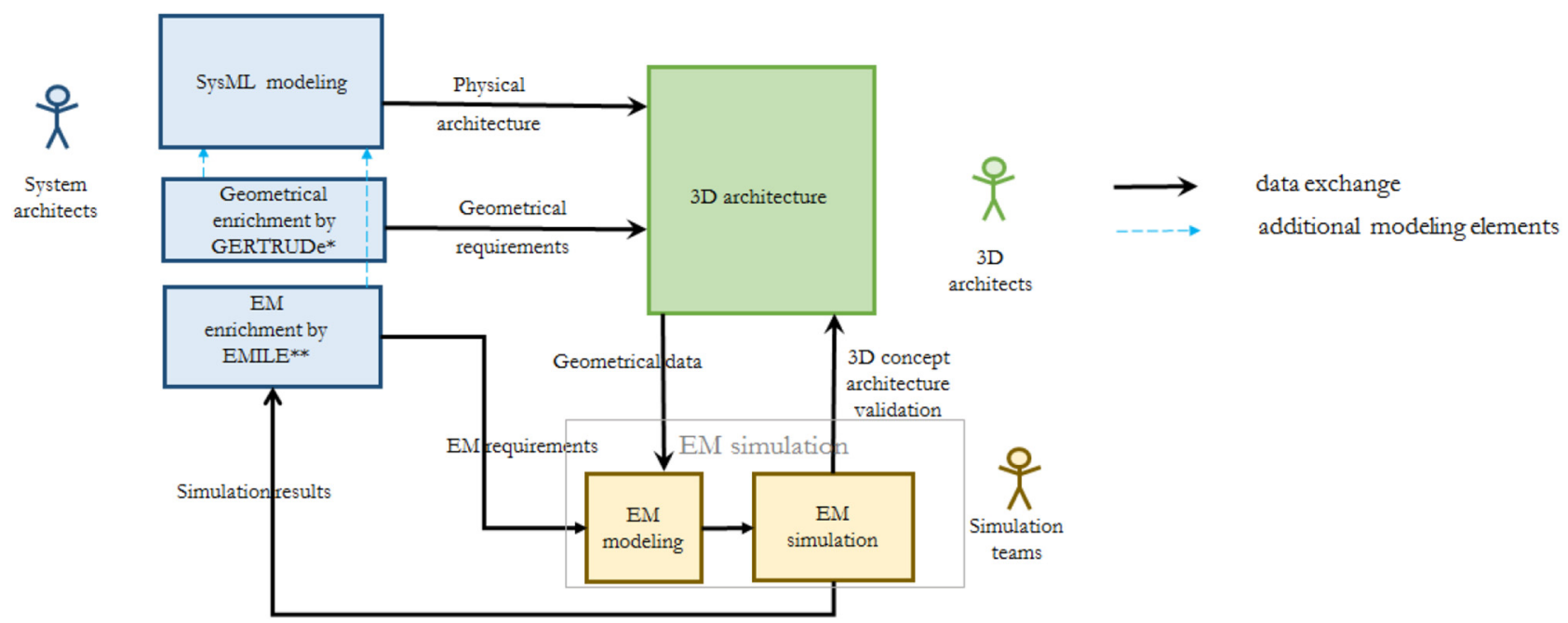

*GERTRUDe Geometrical Extension Related to a TTRS Reference for a Unified Design

**EMILE ElectroMagnetic Interactions Layout Extension

Fig. 2. Integration of EMI in the SAMOS framework.

\subsection{Topological approach for the alien crosstalk identification}

\subsubsection{Introducing crosstalk}

In electronics, crosstalk is any undesired phenomenon whereby signals from one circuit or channel of a transmission system create disturbances on another circuit or channel. Alien crosstalk is the electromagnetic noise that can occur in a cable and is caused by other cables adjacent to the cable of interest as opposed to signals contained in the same cable. Crosstalk can usually occur via electromagnetic fields between conductors caused by undesired capacitive and/or inductive couplings from one circuit or channel to another $[42,43]$.

Inductive crosstalk consists of the following physical phenomenon: the variable magnetic field created by a conductor crossed by a current $I_{S}$, is intercepted by a neighboring conductor behaving as a loop (Fig. 3). A counter-electromotive force therefore appears. This electrical voltage may materialize as a differential or as a common mode (Fig. 4). It is convenient to model the coupling between the two loops by a mutual inductance $\mathrm{M}$ between the two circuits.

Capacitive crosstalk occurs when a variable electric field $\mathrm{E}(\mathrm{t})$ created by the electrical voltage between two conductors couples with a neighboring conductor. As a result, a current appears in this conductor. This electric field coupling is equivalent to a capacitance $\mathrm{C}_{\mathrm{m}}$ that generates the same current through the circuit (Fig. 5). The generated current materializes as a differential or as a common mode in the victim circuit.

\subsubsection{Process overview}

As EM couplings occur between E/E components, the process begins with the description of the corresponding

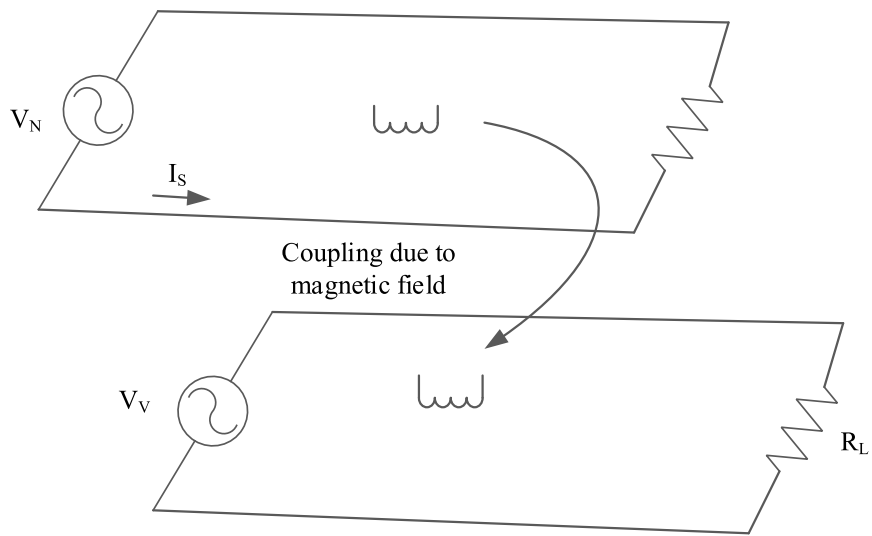

Fig. 3. Inductive crosstalk.

$\mathrm{E} / \mathrm{E}$ physical architecture, which is represented by an electrical diagram, including the equivalent impedance of each component and related circuit voltages (Fig. 6).

Next, as any component may act as a source or victim with relation to another nearby component, we then perform the topological modeling corresponding to the $\mathrm{E} / \mathrm{E}$ physical structure, in order to identify these physical interactions. As imposed nominal physical interactions between $\mathrm{E} / \mathrm{E}$ components occurring in an electrical circuit are based on the currents flowing in cables, we represent these components' interactions (supported by cables) through three topological views: a simplified electrical schematic for the topological modeling (Fig. 7a), a topological graph (Fig.7b), and an Equipment-Cable interaction matrix (Fig. 8) as the physical interaction here is supported by the cables. This matrix, based on the electrical physical structure, named Electrical Matrix, is the transposed incidence matrix including the current flow direction. 


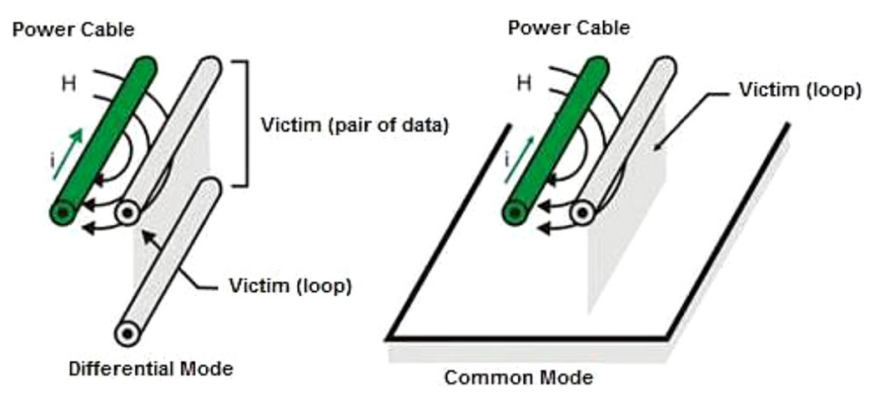

Fig. 4. Inductive coupling modes [54].
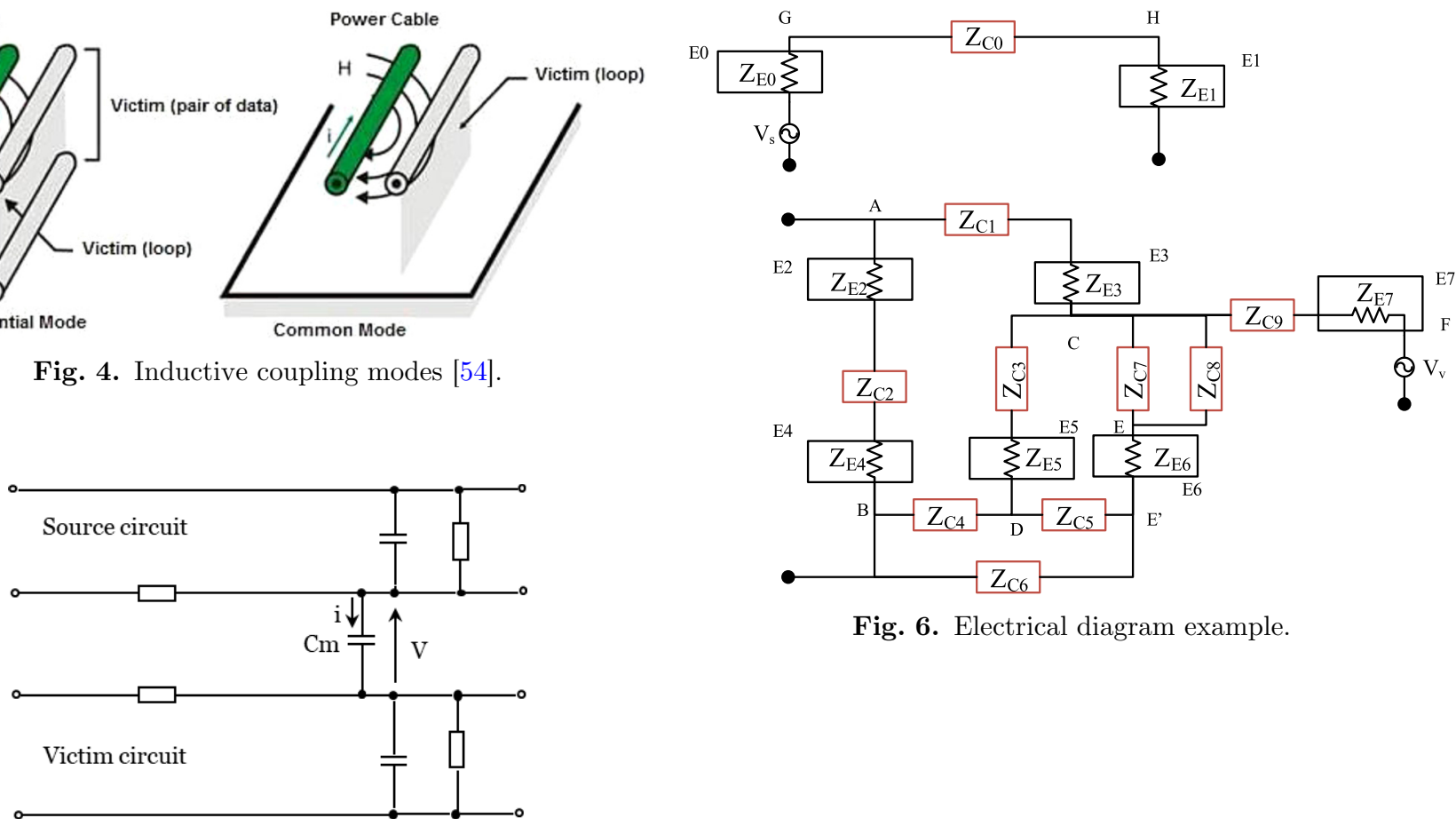

Fig. 6. Electrical diagram example.

Fig. 5. Capacitive crosstalk.
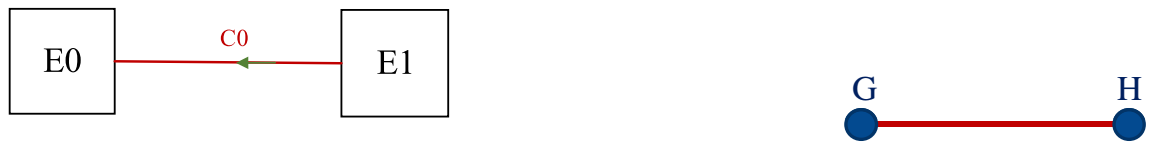

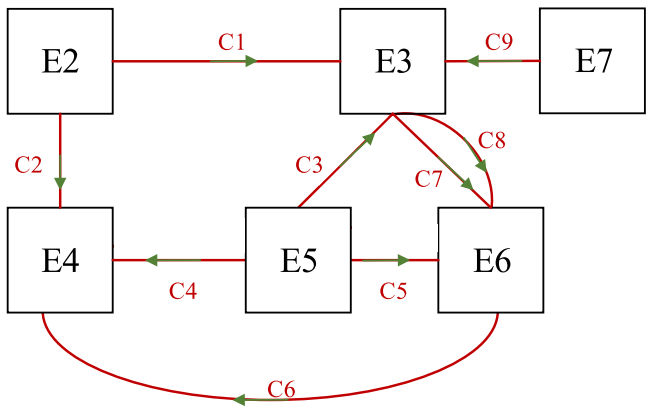

a)

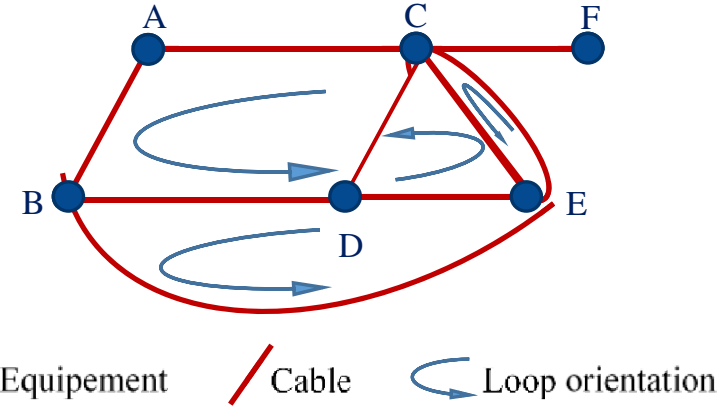

b)

Fig. 7. (a) Simplified E/E physical architecture for the topological modeling. (b) Corresponding topological graph with edges and vertices.

The analysis of such topological graphs and matrixes allows for the identification of the potential EMI disturbances to be considered in the quantitative assessment.

Regarding the crosstalk coupling, be it inductive or capacitive, it requires a victim (current) loop. Thus, our topological approach is first based on the current loops' identification in order to detect the potential crosstalk victims. Then, for each victim/loop recognized, its possible aggressors can be identified, before classifying the corresponding coupling as inductive or capacitive crosstalk. This qualitative evaluation process is generic for all crosstalk types, whatever their coupling mode (common or differential).

\subsubsection{Victims/loops identification}

The first step is the identification of all current victim loops, from the topological graph and the corresponding matrix ME (Fig. 8). 


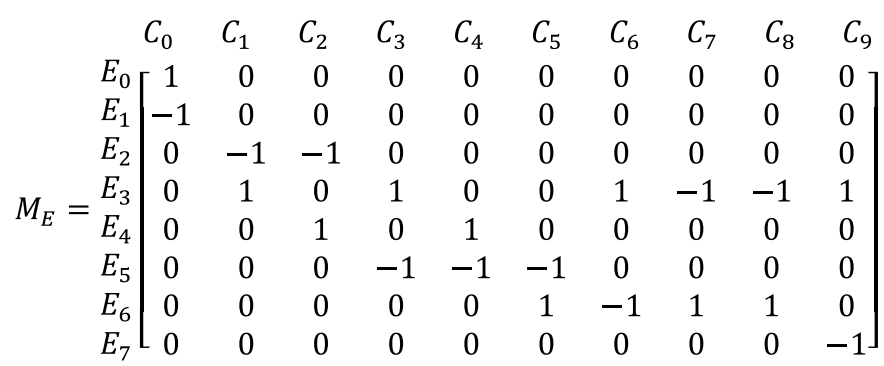

Fig. 8. Electrical matrix (ME) corresponding to the topological graph of the E/E physical architecture.

\begin{tabular}{|c|c|c|c|c|c|c|c|c|}
\hline & $A$ & $B$ & $C$ & $D$ & $E$ & $F$ & $G$ & $H$ \\
\hline $\overrightarrow{A B}$ & {$[-1$} & 1 & 0 & 0 & 0 & 0 & 0 & 0 \\
\hline $\overrightarrow{B D}$ & 0 & -1 & 0 & 1 & 0 & 0 & 0 & 0 \\
\hline $\overrightarrow{C D}$ & 0 & 0 & -1 & 1 & 0 & 0 & 0 & 0 \\
\hline $\overrightarrow{A C}$ & -1 & 0 & 1 & 0 & 0 & 0 & 0 & 0 \\
\hline$=\overrightarrow{C E}$ & 0 & 0 & -1 & 0 & 1 & 0 & 0 & 0 \\
\hline $\overrightarrow{D E}$ & 0 & 0 & 0 & -1 & 1 & 0 & 0 & 0 \\
\hline $\overrightarrow{C F}$ & 0 & 0 & -1 & 0 & 0 & 1 & 0 & 0 \\
\hline $\overrightarrow{G H}$ & 0 & 0 & 0 & 0 & 0 & 0 & 1 & -1 \\
\hline $\overrightarrow{B E}$ & 0 & -1 & 0 & 0 & 1 & 0 & 0 & 0 \\
\hline $\overrightarrow{C E}$ & 0 & 0 & -1 & 0 & 1 & 0 & 0 & 0 \\
\hline
\end{tabular}

Fig. 9. Incidence matrix $\delta^{0}$ between edges and vertices.

Physically, crosstalk occurs when the three following conditions related to the number of current loops $\left(N_{c l}\right)$, the number of cables $\left(N_{c}\right)$ and the number of pieces of equipment $\left(N_{E}\right)$ are met: $N_{c l} \geq 1, N_{c} \geq 3, N_{E} \geq 2$.

The mathematical formula providing the set of current loops $\mathfrak{B}_{m}$ contained in the topological graph [44], is based on the elements of the corresponding $\mathrm{M}_{\mathrm{E}}$ matrix (between the $E_{m}$ pieces of equipment and the $C_{n}$ cables) and is detailed in equation (1):

$\mathfrak{B}_{m}=\left\{\begin{aligned} \operatorname{sign}\left(C_{i}, E_{k}\right) & =\operatorname{sign}\left(C_{j}, E_{l}\right) \\ \left(E_{k}, E_{l}, C_{i}, C_{j}\right) \backslash & =-\operatorname{sign}\left(C_{i}, E_{l}\right) \\ & =-\operatorname{sign}\left(C_{j}, E_{k}\right) \\ & \neq 0\end{aligned}\right\}$

$$
\text { With } i, j=\{0 \ldots n\} ; k, l=\{0 \ldots m\} ;(k \neq l) \text { and }(i \neq j)
$$

We therefore propose a methodology to systematize the identification of these loops through a topological analysis. This automated identification process first consists of integrating the connectivity data resulting from the topological

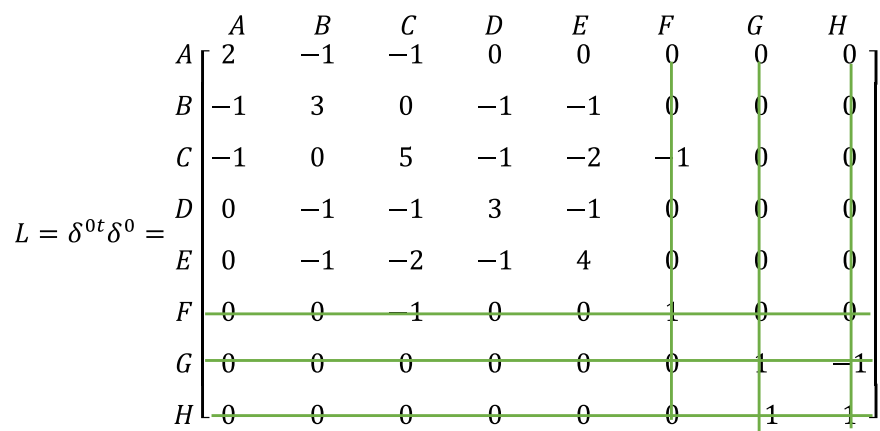

Fig. 10. Laplacian matrix $\delta^{0 t} \delta^{0}$ of vertices with their degrees at the diagonal.

graph (Fig. 7b). Then, the incidence matrix $\delta^{0}$, which represents the relationships between the two classes of objects: edges and vertices, is calculated (Fig. 9).

Next the Laplacian/admittance matrix $L=\delta^{0 t} \delta^{0}$ (Fig. 10), using the topological graph is computed (Fig. 7b). This matrix is defined by the Kirchhoff law, where the vertices' degrees are identified on its diagonal. In fact, the different loops will be identified by iteratively selecting the lowest degree vertices, and removing them from the $\mathrm{M}_{\mathrm{E}}$ before the next iteration.

In practice, the first computational iteration consists of excluding the 1-degree vertices (and related rows and columns), which correspond to vertices with a unique edge, and are then considered as "dead" vertices. However, in the case of the modeling of an antenna, which in theory would be modeled by a 1-degree vertex, we have chosen to describe it in the $\mathrm{M}_{\mathrm{E}}$ as a fictive loop. Finally, in the Laplacian matrix example, the $\mathrm{F}, \mathrm{G}$ and $\mathrm{H}$ vertices will be removed.

For each following iteration, a newly reduced Laplacian matrix with updated vertex degrees taking into account the removed vertices must be evaluated. This process is completed with the identification of the loops related to the 2 -degree vertices. For each analyzed vertex, the adjacency of the connected vertices allows for the definition of the corresponding loops. The whole process allows for the identification of the existing victim loops of 2,3 or 4 vertices (as explained in the Fig. 11 and Fig. 13).

Finally, all identified current loops are put into a global matrix Mat $_{\text {global }}$ as column inputs (connectivity matrix). The rows of this matrix are arbitrarily chosen to define all the edges. Then for each identified victim loop its orientation is arbitrarily chosen (Fig. 7b). Thus, the -1 / +1 value signs in the $M a t_{\text {global }}$ matrix are defined to be consistent with the fixed orientation (Fig. 12).

In our case, we are focusing on simply finding all existing loops without any electromagnetic consideration. For example, the identified loop named ABDC is a loop of 4 vertices connected with the following edges $(\overrightarrow{A B}, \overrightarrow{B D}, \overrightarrow{C D}$ and $\overrightarrow{A C})$.

The following algorithm (Fig. 13) synthesizes the methodology for identifying current victim loops composed of 2,3 or 4 vertices according to their adjacency conditions (Fig. 10). It has been implemented in Matlab to provide an automated identification process. 


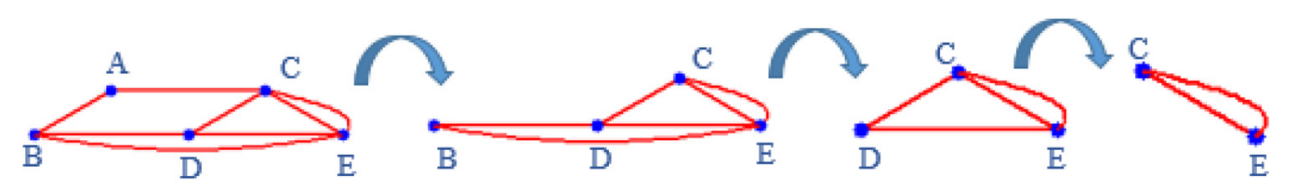

Fig. 11. Evolution of the topological graph over the successive iterations during the loops identification process.

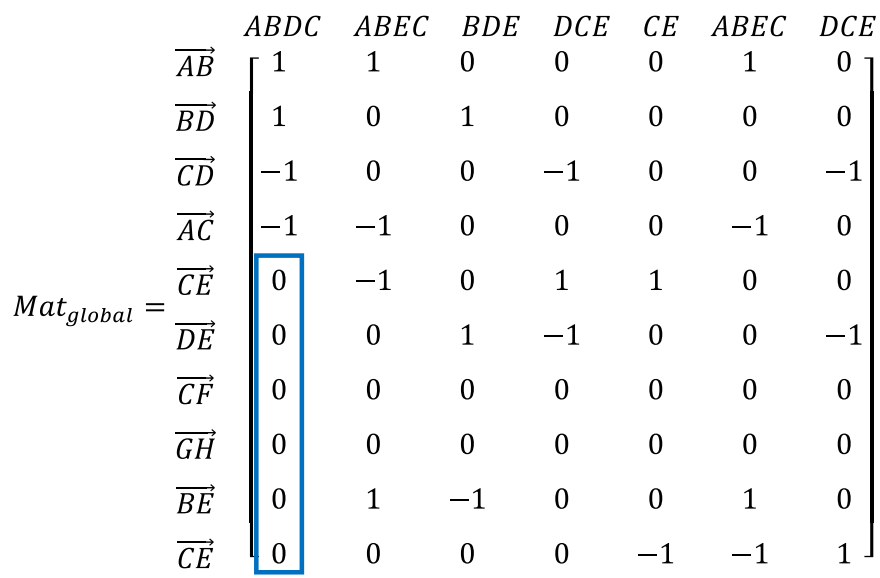

Fig. 12. Global matrix $M a t_{\text {global }}$ of the identified current loops according to the oriented edges.

\subsubsection{Aggressors identification}

For each identified current loop, we identify the corresponding potential aggressors in the system's E/E architecture. In fact, focusing on the crosstalk, potential aggressors are all the edges that are both crossed by a current and do not belong to the specific victim loop.

When considering the global matrix, potential aggressors of each victim loop (i.e. a given column) correspond to the edges (row values) whose values equal 0 (Fig. 12). For example, for the identified current loop named ABDC, all possible source edges are those corresponding to the 0 values in the blue frame in Figure 12.

\subsubsection{Inductive and capacitive crosstalk identification}

In order to determine the potential aggressors respectively related to inductive and capacitive crosstalks, some complementary physical (electrical) information on the electrical parameters to be added are needed, as attributes, on vertices and edges. So, on one hand the inductive coupling is defined by the presence of a disruptive variable current (3) crossing an "aggressive" cable between two pieces of equipment. On the other hand, the capacitive coupling occurs when there is a variable electric field which is created by an aggressive cable exposed to a variable voltage (4). Next, the following mathematical formulas [44] that describe this complementary physical information have been obtained:

$$
\exists i_{i}(t) \neq 0 \backslash\left\{\left\{C_{i},\left(E_{k}, E_{l}\right)\right\} \neq\{\phi\} \mid\left(E_{k}, C_{i}\right)=-\left(E_{l}, C_{i}\right) \neq 0\right\}
$$

$\exists u_{k}(t) \neq 0 \backslash\left\{\left\{E_{k},\left(C_{i}, C_{j}\right)\right\} \neq\{\phi\} \mid\left(E_{k}, C_{i}\right)=-\left(E_{l}, C_{i}\right) \neq 0\right\}$

\subsection{EMI quantitative evaluation}

The next step consists of quantitatively evaluating the 3D architecture in inductive crosstalk based on both the physical and the geometrical parameters of components (source/victim cables). The quantitative assessment is performed by the use of mathematical formulas based on the physical equations of the topological structure, in order to finally identify and predict the EMI between E/E components of a given 3D architecture.

Through an analytical method, the victim voltage of the decibel transfer function for each edge can be plotted in function of the frequency. This curve gathers the tension of one of the identified current loops (here ABDC) at the component terminals in accordance with their position.

\section{Application to the EVPT scenario}

\subsection{Scenario description}

This new approach is illustrated using an Electric Vehicle Power Train (EVPT) architecture where several potential EMIs may emerge due to the close proximity of the high voltage (HV) cables connecting the HV systems (HV battery and power inverter) and the low voltage (LV) cables connecting the LV systems (such as sensors). The way the cables are arranged generates EM couplings which can be sources of EMI [45] and then cause component malfunctions [46]. Then, these interactions need to be assessed to subsequently predict the EMC risks within the system. Figure 14 shows a description of an EVPT on a schematic layout. The scenario presents several potential EMI, but we will describe in this section only the cable to cable interaction, and notably the alien crosstalk, as presented in this figure.

\subsection{EM requirements modeling of the EVPT scenario}

In accordance with a MBSE approach, the evaluation of EMI couplings relates to some components and environment properties, and quantitative results have to be compared to acceptable EM limit values. Therefore, the system architects need to first specify all these requirements and EM modeling in a familiar environment (based on the SysML language), before proceeding to qualitative and quantitative evaluations. For this purpose, we have developed the EMILE SysML extension to support the EM modeling in SysML language, which is detailed in another paper [47]. This extension supports the collaboration between system architects and EM experts, by firstly integrating geometry-based EM modelling in order to formalize EM constraints in the system model, and 


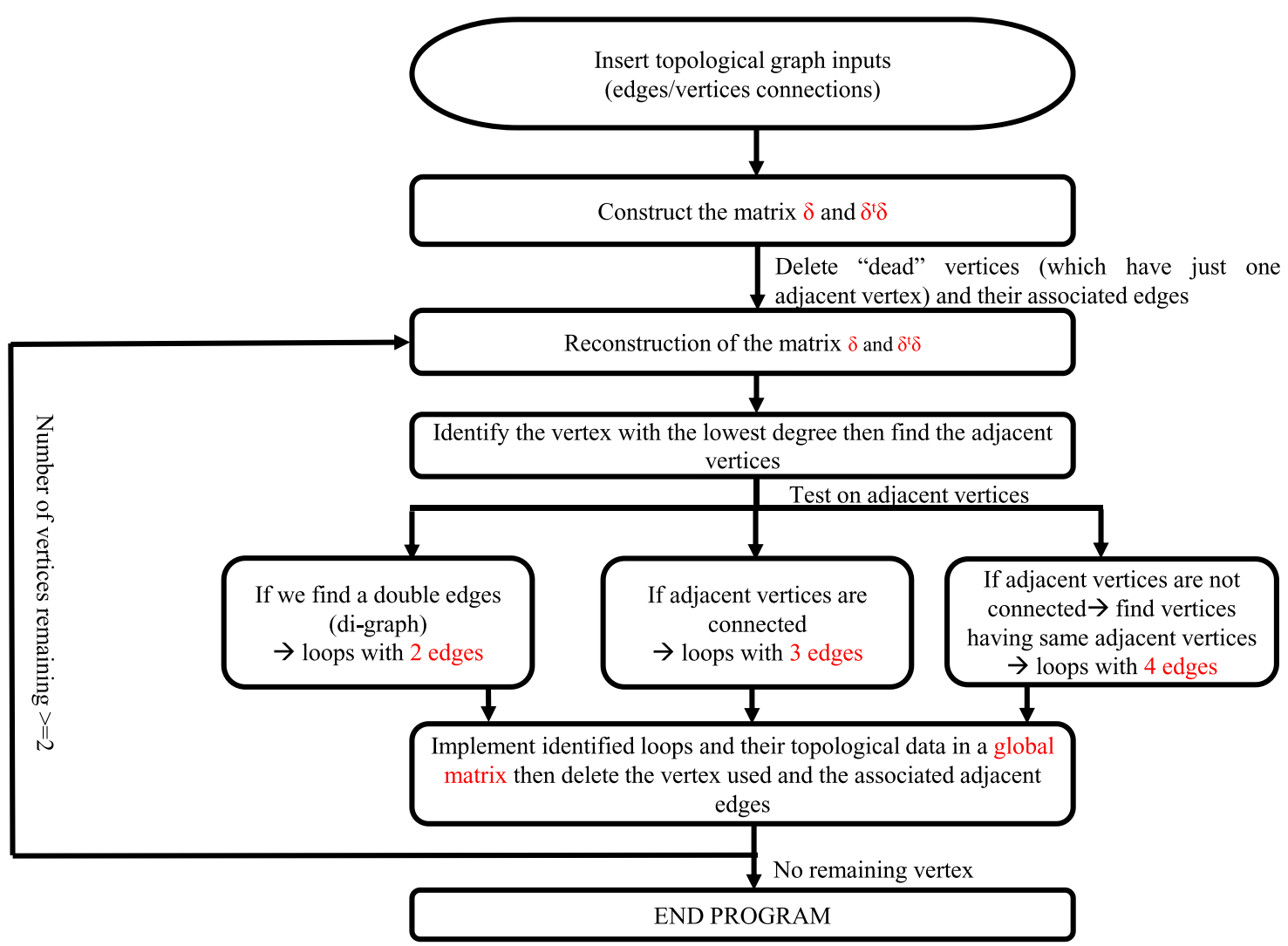

Fig. 13. Victim loops identification process.

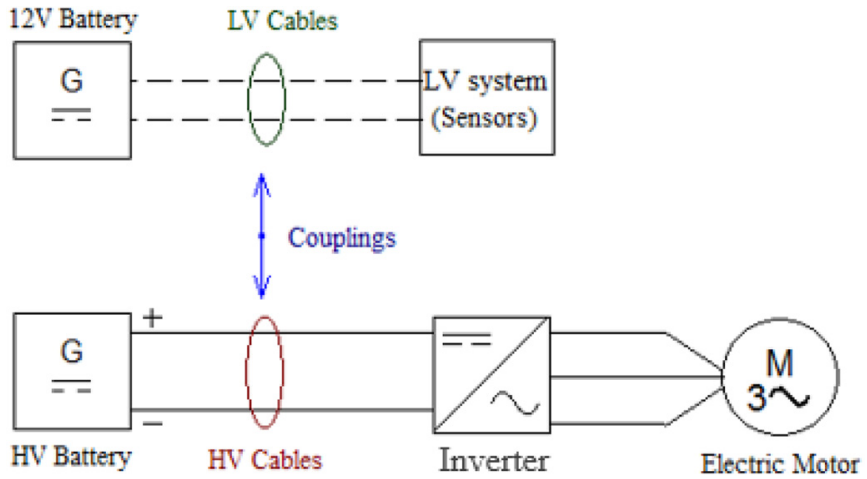

Fig. 14. Schema of the EVPT architecture.

secondly by allowing the simulation configuration to be specified in the system model (through a customized GUI) to transcribe a request from the system architect to the EMC experts. This part mainly concerns our first contribution and is only partially reused in this paper in order to be able to specify all the EM and 3D requirements and modeling defined by the system architects. In this present paper, our main contribution addresses the early EMI qualitative and quantitative assessment of concept architecture. Indeed, the combination of EM modeling and topological analysis in the MBSE SAMOS approach enables support of the assessment process of electromagnetic interference (EMI) for the relevant choice of a 3D physical architecture.

During the conceptual design phase, the process of EMI disturbances assessment must previously define, in accordance with the customers' needs, the EM requirements based on the EMC experts' proficiency and standards. These requirements include EM properties of components, limit values specified by standards, physical solicitations, minimal and maximal permissible limits, and finally, geometrical information (components' geometry and relative positioning constraints).

Thus, the EM requirements of the EVPT scenario include components' geometrical information (shape, dimensions, positioning constraints), their electrical solicitations (frequency, voltage, current) and physical properties (emission or reception properties, material properties). These initial requirements are derived from the available literature on the EVPT components and electrical features [42], which have been estimated when unavailable. These EVPT components' data are synthesized in Table 1.

Next, these requirements have been formalized within the SAMOS platform, in the SysML environment, through the GERTRUDe SysML extension for geometrical information modeling (Fig. 15) and the EMILE SysML extension for the EM modeling (Fig. 16).

An extract of these requirements is shown in a Requirement Diagram (Fig. 17). 
Table 1. EVPT scenario initial requirements.

\begin{tabular}{|c|c|c|c|}
\hline \multicolumn{3}{|l|}{ Parameter } & Value \\
\hline \multicolumn{3}{|l|}{ Nominal HV voltage $\left(V_{s}\right)$} & $V_{s}=[46 \mathrm{~V}, 58 \mathrm{~V}]$ \\
\hline \multicolumn{3}{|c|}{ Immunity power of victim components } & $P_{c}=18 \mathrm{dBm}=63 \mathrm{~mW}$ \\
\hline \multicolumn{3}{|l|}{ Maximal HV intensity } & $I_{s \max }=360 \mathrm{~A}$ \\
\hline \multicolumn{3}{|l|}{ Nominal frequency range } & $\mathrm{f}=[100 \mathrm{~Hz}, 1 \mathrm{MHz}]$ \\
\hline \multicolumn{3}{|l|}{ Limit victim voltage } & $V v_{\text {limit }}=0.2 \mathrm{~V}$ \\
\hline \multirow[t]{2}{*}{ Geometrical parameters } & $\varnothing$ & $\phi_{i H V \text { cable }} \approx 8 \mathrm{~mm} \quad \phi_{i L V \text { cable }} \approx 4 \mathrm{~mm}$ & $\mathrm{HV} / \mathrm{LV}$ cable diameter \\
\hline & $\mathrm{L}$ & $L_{\mathrm{HV} \text { cable }}=L_{\mathrm{LV} \text { cable }} \approx 2 \mathrm{~m}$ & $\mathrm{HV} / \mathrm{LV}$ cable length \\
\hline
\end{tabular}

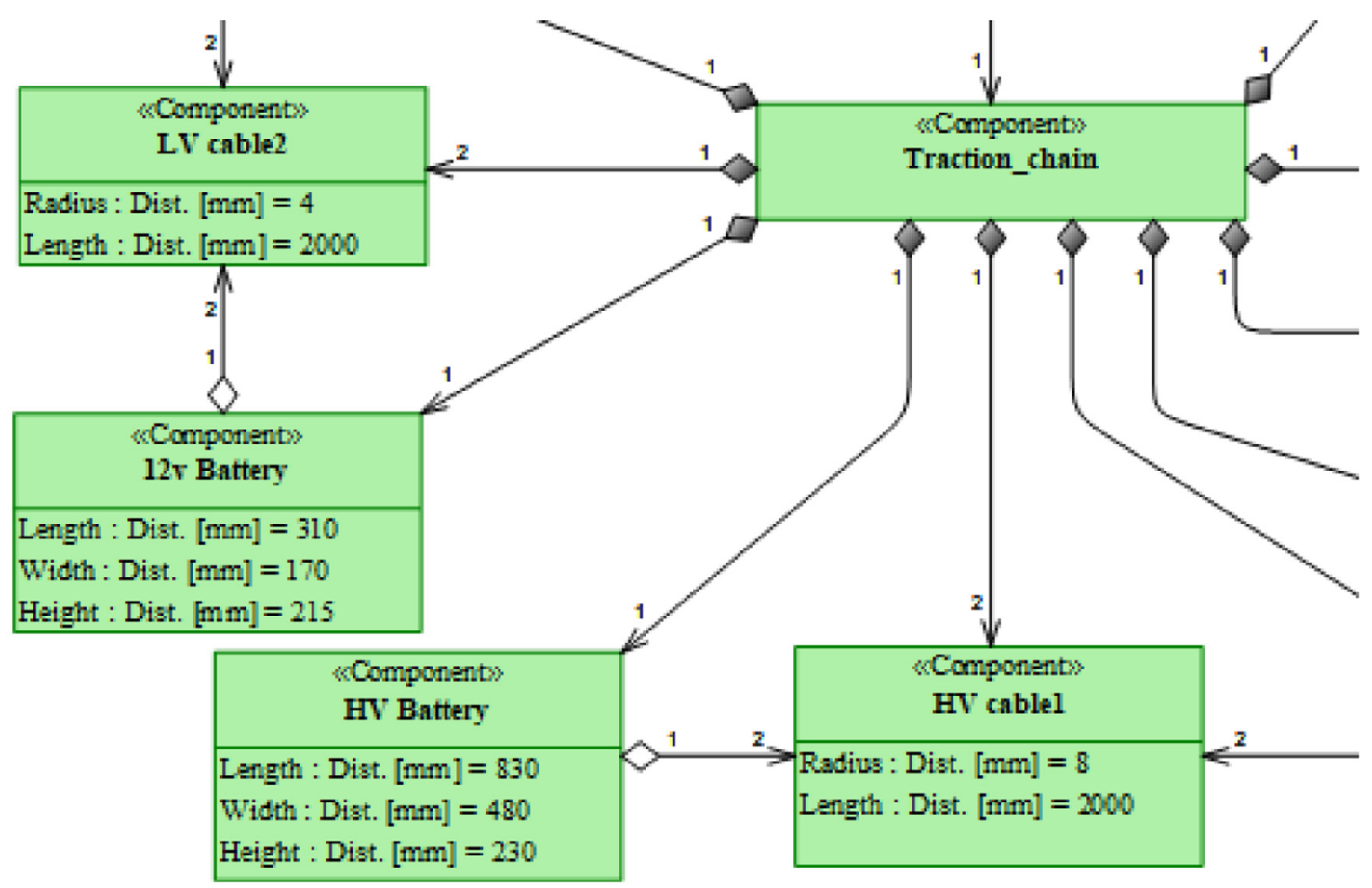

Fig. 15. EVPT geometrical data requirements example with GERTRUDe.

\subsection{Alien crosstalk identification of the EVPT scenario}

In this section, we detail the modeling process associated with the identification of a specific EMI which can appear in the EVPT scenario, i.e. the alien crosstalk.

As described in the previous section (Sect. 3.2), the topological approach to the EMI identification starts with the electrical diagram including all the $\mathrm{E} / \mathrm{E}$ components likely to be EM interactive. The EVPT electrical diagram including the equivalent impedance of each component is represented in Figure 18.

This electrical diagram of the EVPT scenario has been modeled in SysML by an internal block diagram (ibd) in order to be integrated into the SysML environment of SAMOS and is shown in Figure 19.
The topological modeling based on this electrical diagram, including the current flows direction in cables and its corresponding topological graph, is represented in Figure 20. The vertices on the topological graph represent the connection between the cables and the pieces of equipment.

Finally, the Electrical Matrix, detailed in Figure 21, is automatically generated by the Matlab developed program.

Then, in accordance with the process described in Section 3.2, the qualitative evaluation starts with the identification of the current loops present in the EVPT scenario. They have been singled out in Figure 22.

For each identified victim loop, the corresponding potential aggressors have been determined (Fig. 23) by 


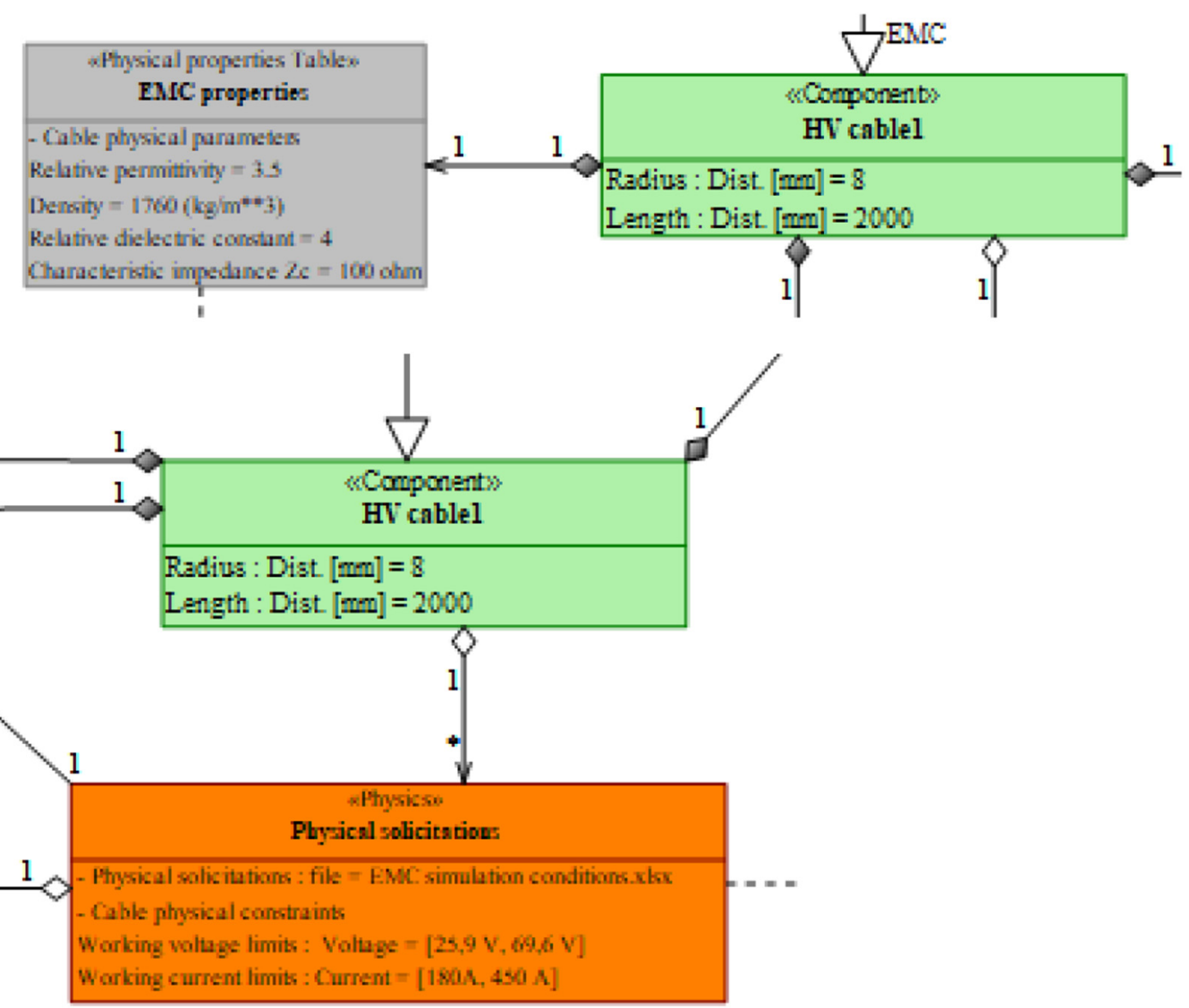

Fig. 16. EVPT physical properties and solicitation example with EMILE.

\begin{tabular}{|c|c|c|}
\hline req Requirement Diagram [EMC Requirements & & \\
\hline & «requirement» & \\
\hline & Business rules requirements & \\
\hline$\Lambda$ & $\Lambda$ & A \\
\hline «refine» & ; refine» & irefine» \\
\hline $\begin{array}{l}\text { «Quantified Requirement» } \\
\text { HV Inverter emission level }\end{array}$ & $\begin{array}{l}\text { «Quantified Requirement» } \\
\text { Minimal distance }\end{array}$ & $\begin{array}{l}\text { "Quantified Requirement» } \\
\text { LV Sensor immunity level }\end{array}$ \\
\hline $\begin{array}{l}\text { Description } \\
\text { The system must withstand the HV } \\
\text { inverter emission level }\end{array}$ & $\begin{array}{l}\text { Description } \\
\text { The distance between the inverter cable } \\
\text { and the sensor cable should exceed }\end{array}$ & $\begin{array}{l}\text { Description } \\
\text { The system must fullfill the LV Sensor } \\
\text { immunity level }\end{array}$ \\
\hline $\begin{array}{c}\text { Attribute } \\
\text { «Attribute» Emission spectrum : Array }\end{array}$ & the minimal distance $=\mathrm{dmin}$ & \begin{tabular}{l}
\multicolumn{1}{c}{ Attribute } \\
«Attribute» Immunity spectrum : \\
Array
\end{tabular} \\
\hline
\end{tabular}

Fig. 17. Extract of the requirements related to the EM modeling based on a geometrical constraint in a Requirement Diagram.

selecting the edges that are both crossed by a current and do not belong to this loop.

Finally, to distinguish inductive and capacitive crosstalk, based on the physical solicitation described in the initial requirements, in our example we consider disruptive variable currents in the $\mathrm{HV}$ cables ( $\mathrm{C} 0$ and $\mathrm{C} 2)$. Then we identify each possible inductive crosstalk between these $\mathrm{HV}$ cables and its related victim loop (ABDC), in order to thereafter quantitatively evaluate the corresponding EMI disturbances.

\subsubsection{Updating the system model}

As we have identified inductive crosstalk as potential EMI, the EM modeling with EMILE can be updated to describe this physical interaction so it can be taken into 

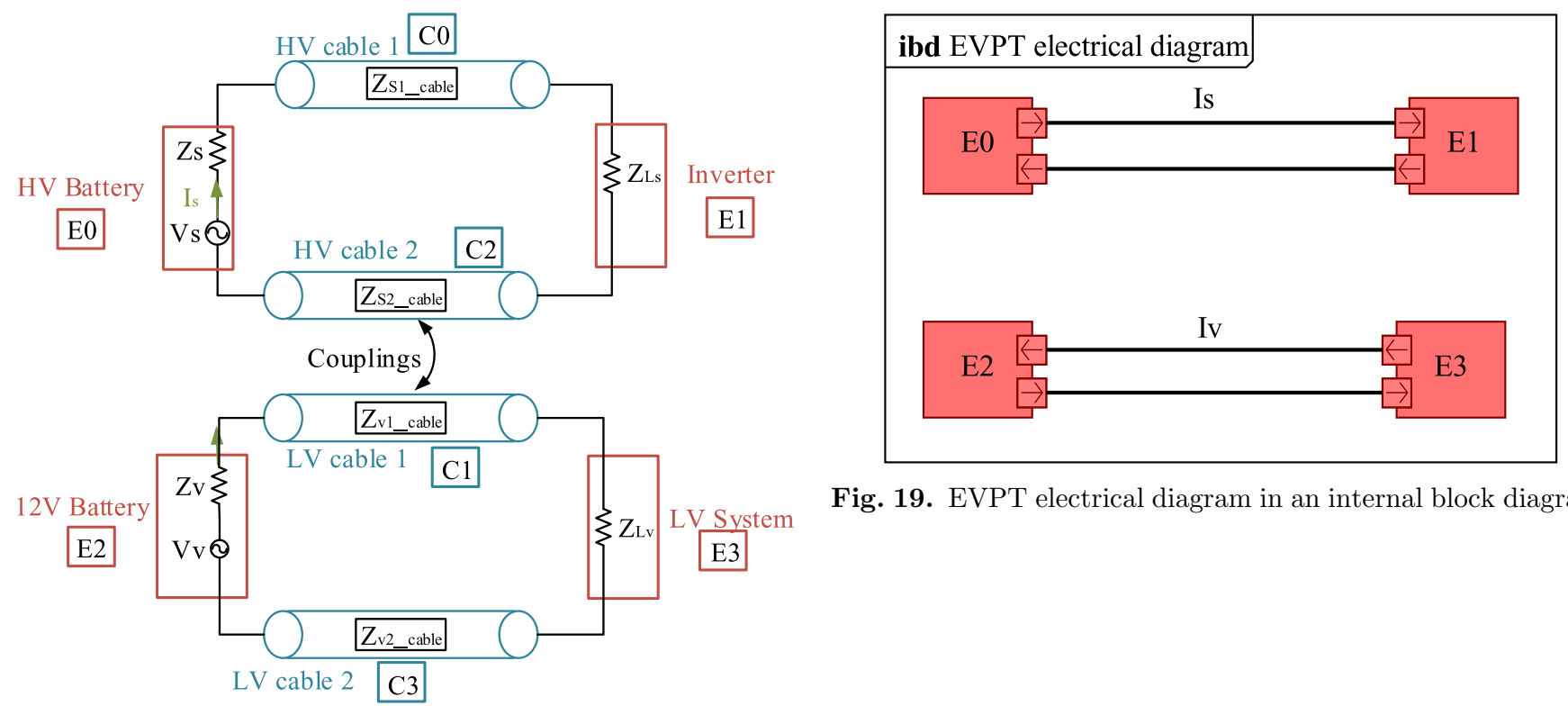

Fig. 19. EVPT electrical diagram in an internal block diagram.

Fig. 18. EVPT electrical diagram.
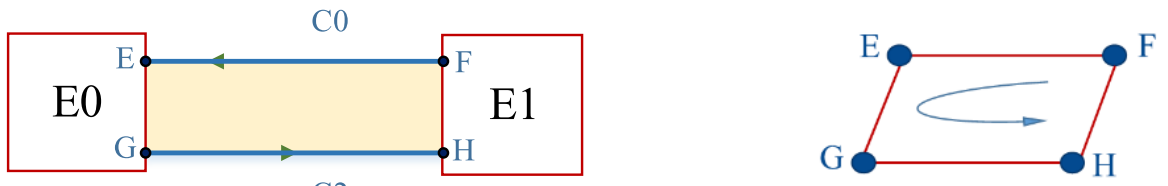

$\mathrm{C} 2$
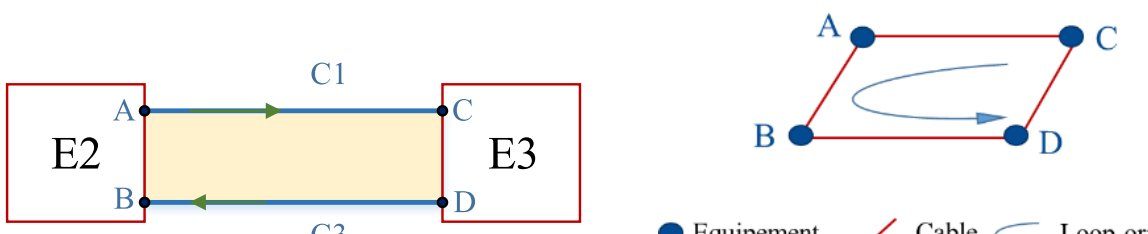

C3

Equipement

Cable $\subset$ Loop orientation

Fig. 20. EVPT topological modeling and graph.

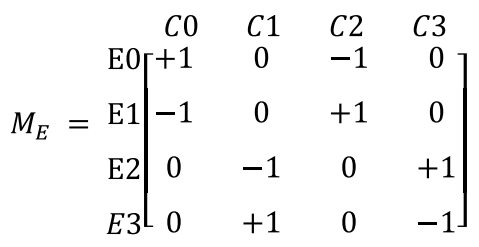

Fig. 21. EVPT electric matrix.

$$
\begin{aligned}
& \text { Loop }= \\
& \text { ABDC } \\
& \text { EGHF }
\end{aligned}
$$

Fig. 22. Identification of the current loops existing in the EVPT scenario in Matlab.

account in the 3D environment of SAMOS. To model the EM interactions between the architecture components, EMILE enriches the internal block diagram (named Physics IBD diagram) with specific EMC ports depending

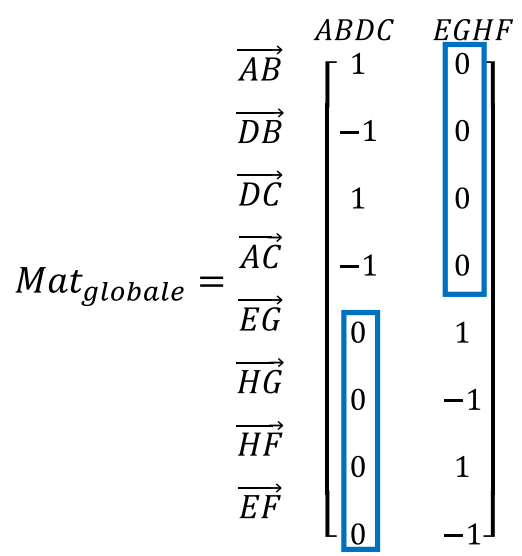

Fig. 23. Identification of the potential aggressors in the EVPT scenario in Matlab.

on the propagation flows involved: RI for radiated interaction, CI for conducted radiation and EI for electrical interaction. 


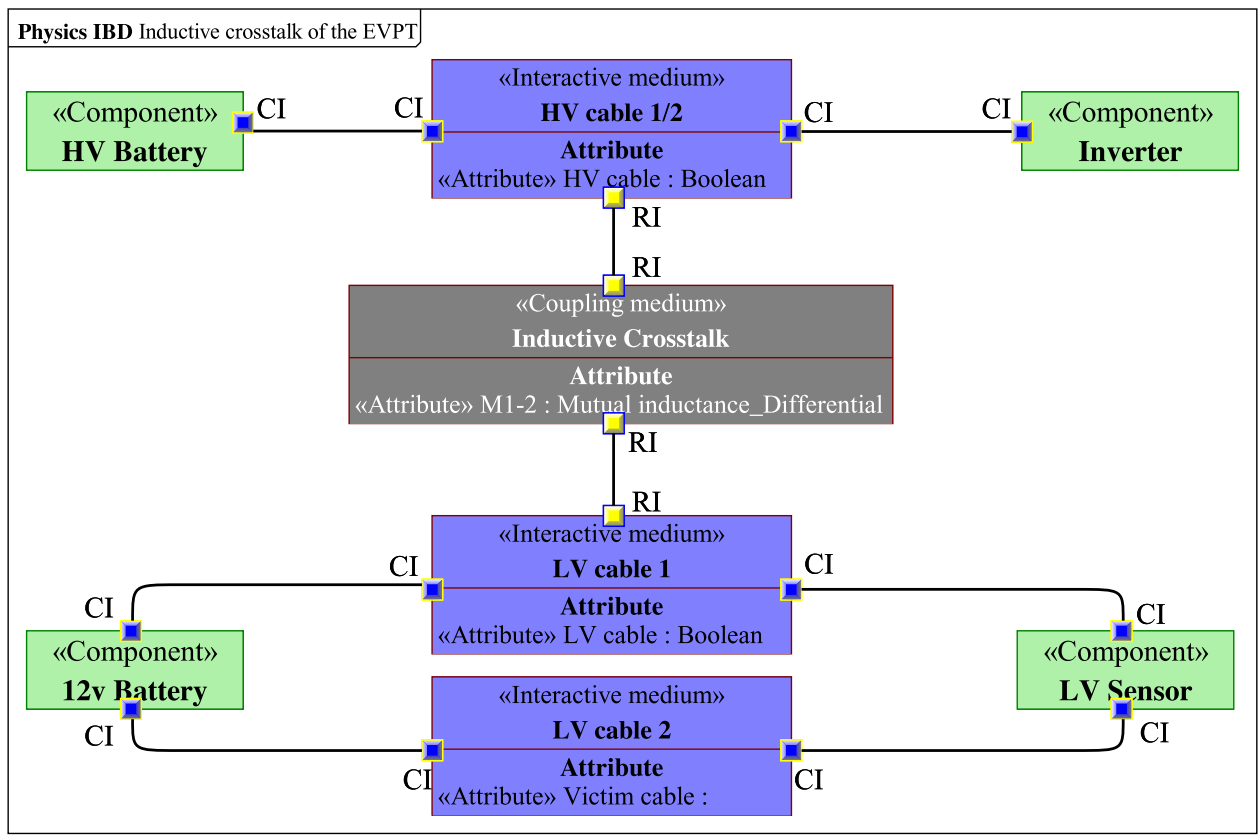

Fig. 24. Physics IBD diagram for the inductive crosstalk description of the EVPT.

For inductive crosstalk in the EVPT scenario, this diagram allows the introduction of the mutual inductance parameter M12 between the two nearby cables (source and victim loop) connected via the RI EMC ports (Fig. 24).

Another diagram has been developed in the EMILE SysML extension: the parametric physics diagram $(P P D)$ to define EMC equation and related parameters' interactions. For the EVPT inductive crosstalk EMI, the mutual inductance equation for the inductive crosstalk related to the cables' geometry and position is described in Figure 25. The links between the two cables and the car body (as we assumed a common mode coupling), declared as being interactive media (interactive components) with the inductive crosstalk coupling medium (containing the EMI behavioral equation), are performed by some specific EMC ports: ports for the physical parameters (red ports) and ports for the geometric parameters (yellow ports).

\subsubsection{EMI disturbances evaluation of the EVPT scenario}

The EMI disturbances quantitative evaluation is based on the analysis of the physical laws describing each coupling type. This step can be performed by different methods, be it analytical or numerical, depending on the experts' preferences, the design phase addressed, and the time constraints. For the EVPT scenario we focus on the analysis of the inductive crosstalk, with two different resolution methods; one (analytical) integrating explicit geometrical parameters (with $\mathrm{d}$ as the distance between the cables of the source and victim loop (assumed the same for the example), and s as the distance between the source loop and the victim loop) as shown in Figure 26; and the other (Kron's) based on a tensorial approach, as detailed in the following sections. We use the same characteristic impedance $\mathrm{Z}_{\mathrm{c}}=100 \Omega$ for both source and victim loops with the same length for source/victim cables $(l=2 \mathrm{~m})$.

\subsubsection{Analytic approach}

An analytical method can be applied to the EVPT scenario, by relying on the physical formulas involved in the inductive crosstalk. This analytical approach consists of the resolution of the Maxwell equations, combined with the discretization and meshes of the identified current loop surfaces, in accordance with the components' geometries and positions.

As the inductive crosstalk appears when a variable current passing through a cable generates a magnetic field $\vec{H}$ in a nearby loop, the first step is to identify all the edges' "sources" powered by a voltage source $V s$ which generates a variable current $I s$. The magnetic induction field $\vec{B}$ emitted in each related victim loop is then described by the Biot-Savart law:

$$
\vec{B}=\mu_{0} \vec{H}=\frac{\mu_{0} I_{s}}{4 \pi} \int_{\text {wire }} \frac{\overrightarrow{d l} \wedge \vec{u}}{r^{2}}
$$

where

$\overrightarrow{d l}$ : vector line element with the same direction as the current $I_{A}$;

$\mu_{0}$ : magnetic constant;

$r$ : distance between the position of $\overrightarrow{d l}$ and the location where the magnetic field is calculated;

$\vec{u}$ : A unit vector in the direction of $r$.

This calculation is carried out in Matlab by summing up all the electromagnetic fields calculated in each point related to a loop discretized area.

Then, from the calculated magnetic field, we can determine the electromotive force $e$ induced in the surface 


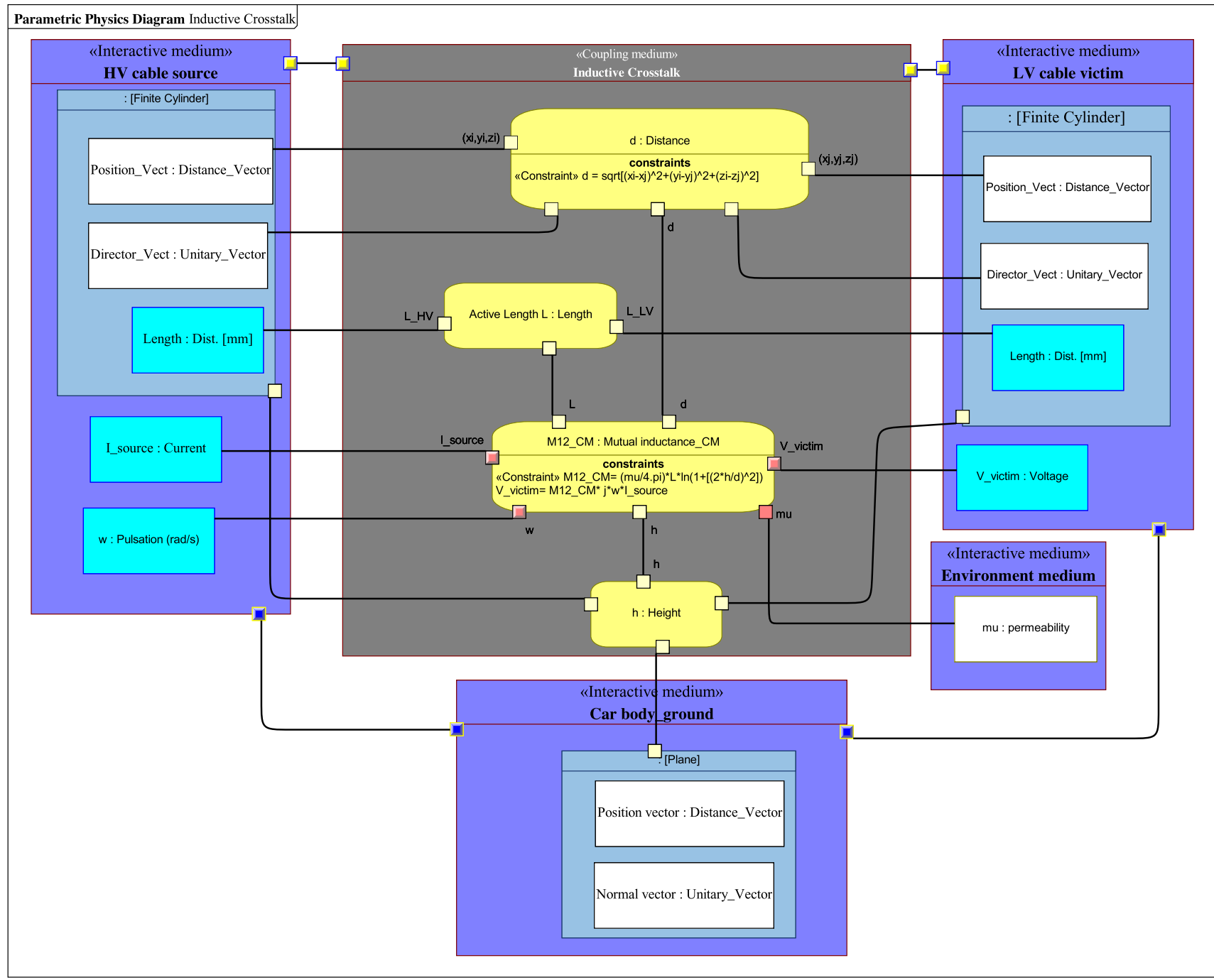

Fig. 25. Parametric physics diagram (PPD) for the definition of inductive crosstalk equation.

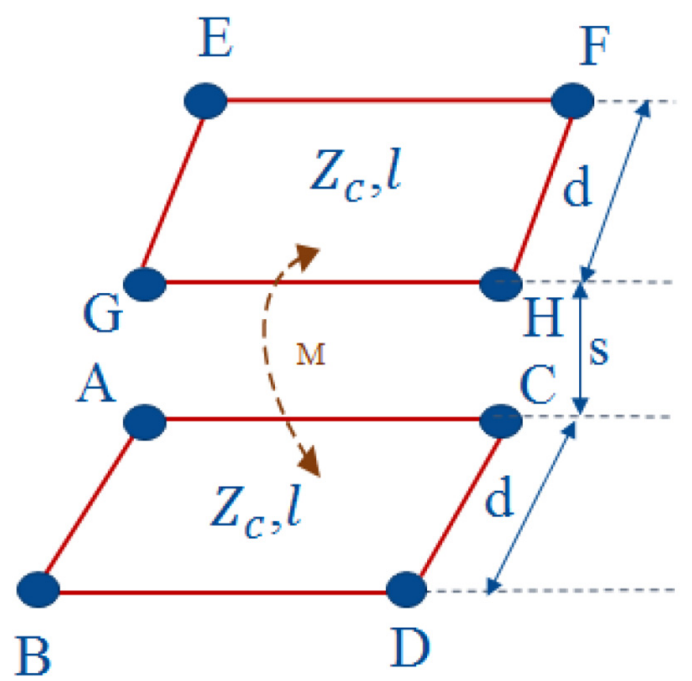

Fig. 26. Geometrical parameters considered for EVPT scenario. loop $S$, using the Lenz-Faraday law, which corresponds to the additional voltage that will be generated in the loop due to the inductive crosstalk:

$$
\begin{gathered}
e=-\frac{d \phi}{\mathrm{d} t}=-\mu_{0} S \frac{d \vec{H}}{\mathrm{~d} t} \cdot \vec{n} \\
\text { with } \phi=\vec{B} \cdot S \vec{n}
\end{gathered}
$$

Assuming that the lengths of the parallel cables constituting the loops, are electrically short $(1<0.25 \lambda$ of the disturbance signal), it can be assumed that the distributed wire-to-wire capacity and the distributed mutual inductance are assimilated to a localized capacity and mutual inductance. Then, each current loop can be defined by a characteristic impedance, a line inductance, and a line capacitance, which are expressed according to 
the relevant cables' geometry and position, based on the following expressions [30,31,40,42,48]:

$$
\begin{gathered}
\text { Characteristic impedance: } Z_{c}=120 \ln \left(\frac{d}{r}\right) \\
\text { Line inductance: } L_{a}=\frac{z_{c}}{v} l \\
\text { Line capacitance: } C_{a}=\frac{1}{z_{c} v} l \\
\text { Line resistance: } R_{a}=\frac{\rho}{s} l
\end{gathered}
$$

with $v$ : propagation speed, $l$ : length of the line, $d$ : distance between the cables, $r$ : cable radius.

Source and victim current loops are coupled by the mutual inductance $M$ expressed by:

$$
M=\frac{\mu_{0}}{4 \pi} \ln \left(1+\left(\frac{d}{s}\right)^{2}\right)
$$

with $\mu_{0}$ : magnetic permeability of free space; $d$ is the distance between the cables of the victim loop; and $\mathrm{s}$ is the distance between the source loop and the victim loop (calculated here at the mid-point of each loop).

Next, we evaluate the induced victim current $I_{v}$, in order to finally calculate the global resulting disturbance voltage $V_{v}$ at the terminals of the victim component in each identified current loop

$$
V_{v}=R_{L} I_{v}
$$

This calculated disturbance voltage, presented in the Figure 28 and Figure 29, allows for evaluation of the EMI risk, by comparing it with the voltage limit $V_{\text {limit }}$ defined for the corresponding component in the previous EM initial requirements.

\subsubsection{KRON's approach}

Maurice provides a topological approach, initially proposed by Kron for electrical circuits [49], to tackle the EMC issues of complex systems [30]. It consists of modeling all the physical interactions between electronic components contained in complex systems, taking into account both the conducting disturbances and radiation couplings (even the nonlinear ones). This approach is thus based on a simplified topological modeling of sub-systems, and on the development of a calculation using any type of model (based on 3D computations, experimental tests, analytical equations, etc.).

In the EVPT scenario the topological modeling is composed of two current loops (named $m_{1}$ and $m_{2}$ meshes) (Fig. 27). Each current loop has two vertices $\left(E_{i}\right)$ and two edges $\left(C_{i}\right)$, including their specified parameters such as

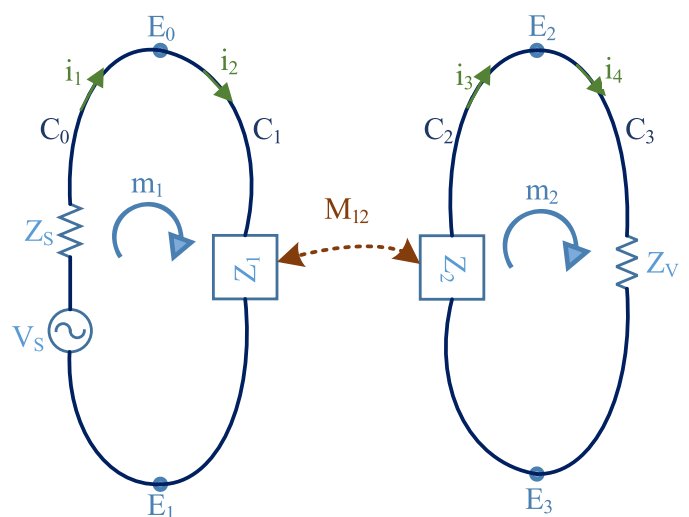

Fig. 27. Topological modeling of EVPT scenario by KRON's method.

their electrical impedances $\left(Z_{i}\right)$. The inductive crosstalk between these two circuits is represented by a mutual inductance $M_{12}$.

The Kron's method can be described in four steps:

- Determining all the edge impedances $Z_{i}$, and the source voltage vector $E_{b}$ involved in the whole system related to the edges' space (Fig. 27):

$$
Z_{e}=\left[\begin{array}{llll}
C 0 & C 1 & C 2 & C 3 \\
Z_{S} & 0 & 0 & 0 \\
0 & Z_{1} & 0 & 0 \\
0 & 0 & Z_{2} & 0 \\
0 & 0 & 0 & Z_{v}
\end{array}\right] ; E_{b}=\begin{aligned}
& C 0 \\
& C 1 \\
& C 2 \\
& C 3
\end{aligned}\left[\begin{array}{l}
V_{S} \\
0 \\
0 \\
0
\end{array}\right]
$$

with

$$
\begin{aligned}
& Z_{S}=R_{S} ; \quad Z_{1}=R_{1}+L_{1} p+\frac{1}{c_{1} p} ; \quad Z_{2}=R_{2}+L_{2} p+\frac{1}{c_{2} p} ; \\
& Z_{v}=R_{v}
\end{aligned}
$$

- Adding the coupling $M_{i}$ (here the mutual inductance) between the edges:

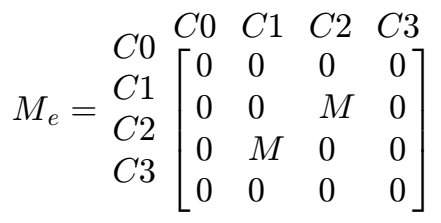

with $M=M_{12} p$

Where $M_{12}=$ the mutual inductance between edge 1 and edge $2 ; p=$ the Laplace operator.

- Defining the connectivity matrix $C_{C \rightarrow m}$ to identify the edges involved in each loop/mesh and to correlate the edges' space with the mesh space;

$$
C_{e \rightarrow m}=\begin{gathered}
C 0 \\
C 1 \\
C 2 \\
C 3
\end{gathered}\left[\begin{array}{ll}
m_{1} & m_{2} \\
1 & 0 \\
0 & 1 \\
0 & 1
\end{array}\right]
$$

- Analyzing the Lagrangian problem in the mesh space, then eventually solving it by resolving these following 


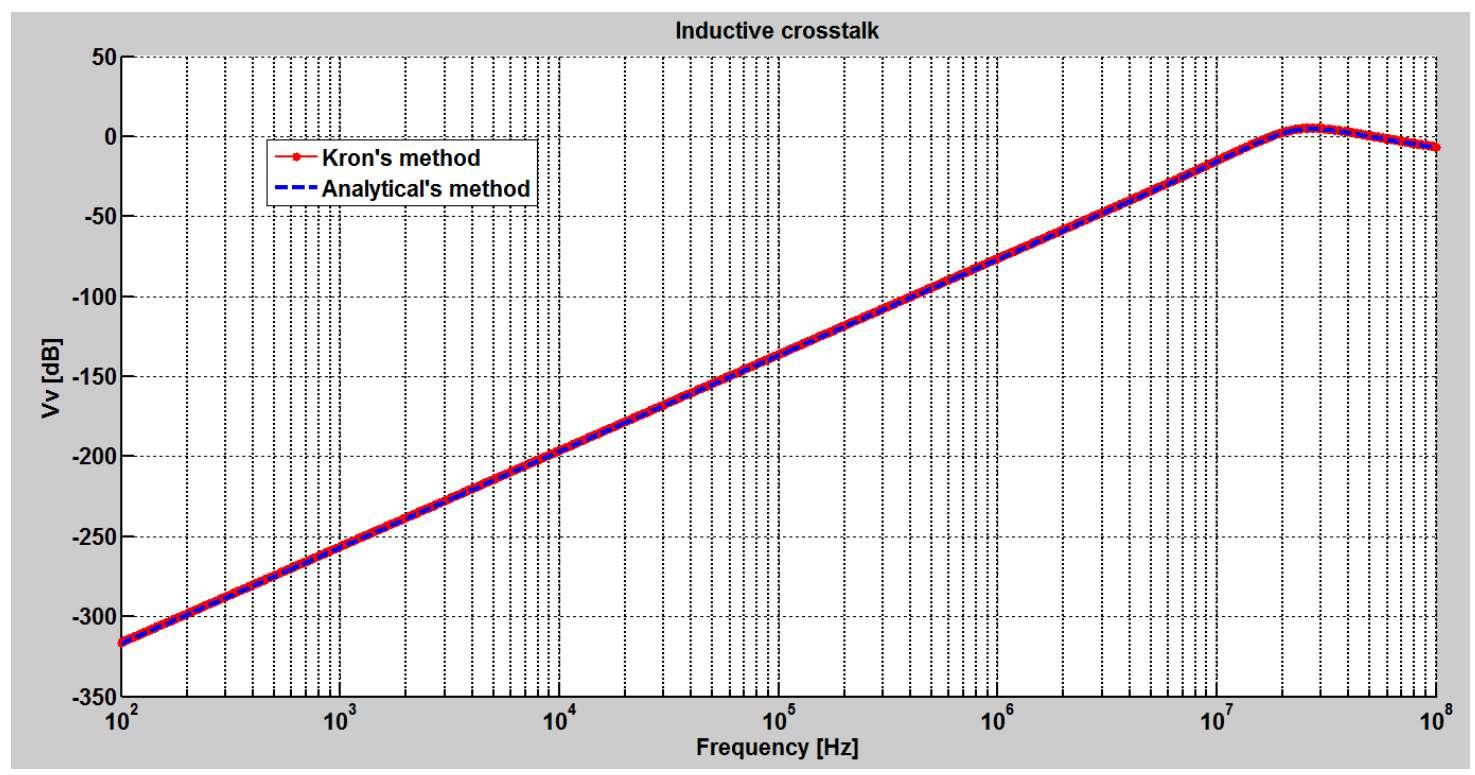

Fig. 28. Victim voltage of the EVPT scenario for analytical and Kron's approaches for constant M and Zc parameters.

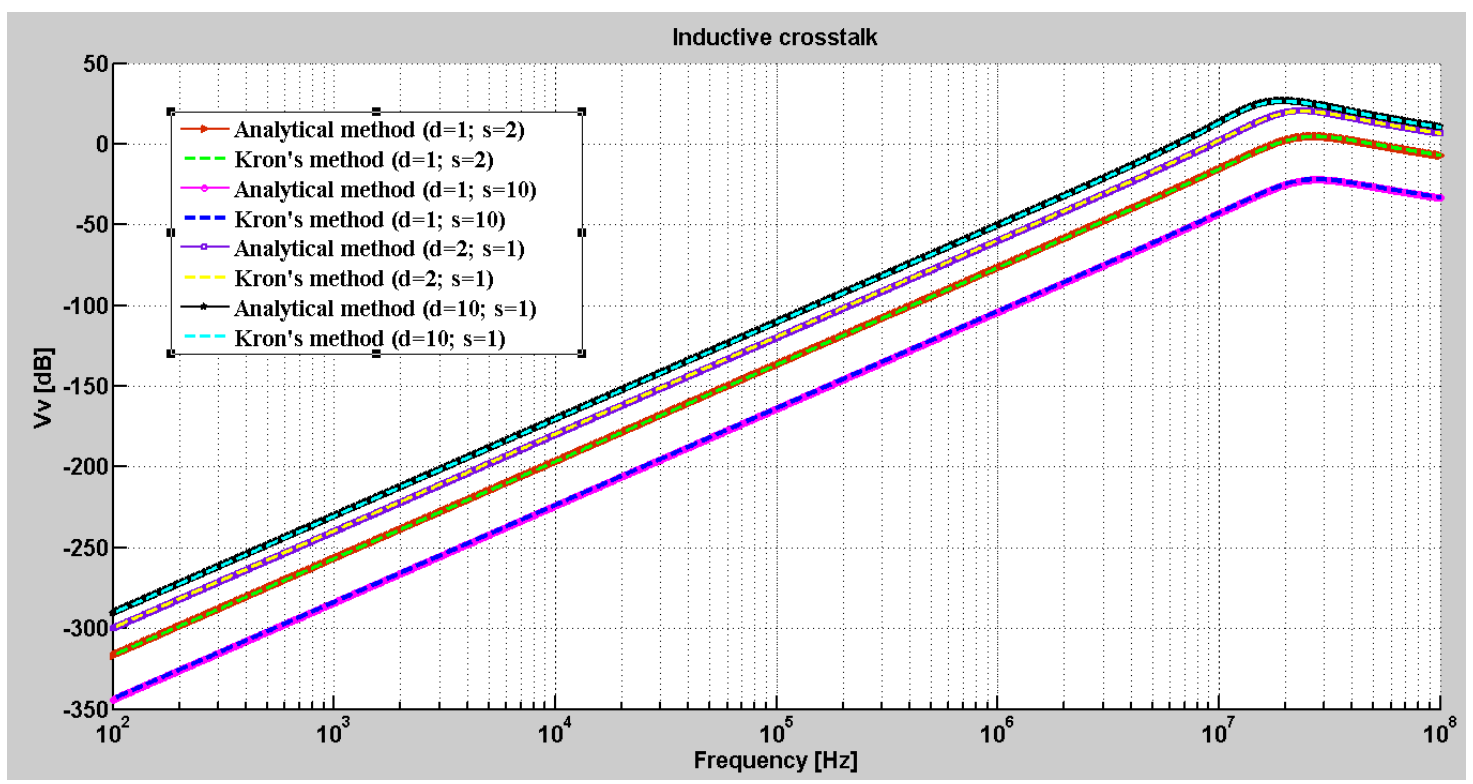

Fig. 29. Victim voltage of the EVPT scenario for analytical and Kron's approaches with different values of d(mm) and $\mathrm{s}(\mathrm{mm})$.

equations [50] with a numerical tool (here Matlab):

$$
\left\{\begin{array}{c}
E_{m}=C_{e \rightarrow m}^{t} \cdot E_{e} \\
Z_{m}=C_{e \rightarrow m}^{t} \cdot\left(Z_{e}-M_{e}\right) \cdot C_{e \rightarrow m} \\
E_{m}=Z_{m} \cdot I_{m} \\
V_{v}=R_{v} \cdot C_{e \rightarrow m}(4,:) \cdot I_{m}
\end{array}\right.
$$

The resolution of the previous equations is shown by the curves in Figure 28 and Figure 29, reflecting the resulting voltage $V v$ at the terminals of the victim component.

\subsubsection{Quantitative results}

We calculate the variation of the magnetic crosstalk using two different methods: the analytical method with the consideration of geometrical parameters $(d, s)$ and the Kron's method (without any explicit geometrical consideration). Figure 28 presents the calculated victim's voltage as a function of the source signal frequency, for both analytical and Kron's approach, based on the same common physical parameters' values: the geometrical parameters' values (for the analytical approach) 
$d=1 \mathrm{~mm} ; r=0.43 \mathrm{~mm}$ and $s=2 \mathrm{~mm}$ and the corresponding calculated characteristic impedance $Z_{c}=100 \Omega$ and mutual inductance $M=2.2310^{-8} \mathrm{H}$.

From these curves, it can be deduced that, based on the same values of the common parameters $\left(\mathrm{M}\right.$ and $\left.\mathrm{Z}_{\mathrm{c}}\right)$, the resulting victim's voltage curves calculated from the two approaches are similar, while the magnetic crosstalk increases with frequency. In fact, at low frequencies, the curves are almost linear and the victim's voltage increases with the frequency, whereas at high frequencies, after a maximum peak (resonance), the curves decrease slowly. This strong resonance appearing in the coupling is situated around $\mathrm{f}=2.510^{7} \mathrm{~Hz}$. As our inverter typically covers a frequency range of $100 \mathrm{~Hz}$ to $1 \mathrm{MHz}$, we can deduce that the resonance of the system is outside the operating range and therefore the operation of the system presents a low risk of EM disturbances in this frequency range.

Then the magnetic crosstalk is calculated when changing geometrical parameters ( $d$ and $s$ ). The resulting calculated voltages are shown in Figure 29.

From the curves of Figure 29 we can deduce that the magnetic crosstalk depends not only on frequency, but also on the relative positioning of cables. The distance d plays then a significant role since it directly determines the area of the loops, and therefore the mutual inductance. Therefore, when varying the distance $\mathrm{d}$ for a constant $\mathrm{s}$, the inductive crosstalk increases. Furthermore, with a constant $\mathrm{d}$, the inductive crosstalk decreases according to the increase of the distance s between the victim loop and source loop. In fact, the further the distance between the source's and victim's loops, the weaker the alien crosstalk coupling.

\section{Discussion and future work}

We have compared our qualitative evaluation approach based on a topology-based process to other existing approaches, such as the minimal spanning tree (MST) method and the depth-first searches (DFS). The main objective of these methods is to minimize the number of connection links by maintaining a single path between all pairs of nodes. The MST method provides a subset of the edges of a connected, edge-weighted, undirected graph. This graph (MST) connects all the vertices together, without any cycles and with the minimum possible total edge weight, so it does not allow the direct identification of cycles (for us physical loops). In parallel, other topological approaches exist in the literature to detect loops (or cycles) in directed and undirected graphs [51,52]. However, the only traditional methods that ensure the detection of cycles within a directed or undirected graph rely on DFS [51,53]. These methods exploit the fact that a graph has a loop/ cycle if, and only if, the DFS method reaches a so-called "back edge" (detected by keeping the list of visited nodes and verifying for rehearsed entries in the list). This approach is quite effective in a limited context, but we have encountered several problems when applying it to our case study topological graph. Indeed, this method could not reach all nodes adjacent to the node already defined with a single path, which requires a longer computation time, or several additional settings in the DFS method. Moreover, it allows only elementary loops to be detected (and not complementary loops). Finally, our topological approach for the quantitative evaluation can identify, from any complex graph with n-nodes already defined, all existing loops, even non elementary ones, while taking into account electromagnetic constraints in the upstream phase.

Regarding the quantitative assessment, many methods are available, depending on the EMC designers' preferences and the availability of the EMC tools at their disposal. Based on Figure 28 and 29, which present the calculated results from two examples of approaches (analytical and Kron's) we have observed that the calculated victim voltage is unchanged regardless of the method of resolution. However, one difference is that the first modeling with the analytical approach based on physical laws explicitly takes into account the geometry and the relative position between components, whereas the analysis by Kron's method uses the electrical equations without any explicit consideration of the geometry and positioning of the components (which are "encapsulated" in the characteristic impedance and mutual inductance values). Therefore, when regarding the SAMOS approach and its implementation in the 3D multi-physical sketcher environment, attention will be paid to identifying physical approaches where geometrical parameters and relative components' positions can be easily extracted/inputted, in order to be able to both compare these parameters with the geometrical and physical requirements of the system architect, and to share them with the 3D architect in the 3D environment). In this way, its compliance with the collaborative process defined for the evaluation of 3D architecture under electromagnetic constraints will be ensured.

Our future work will address the implementation of this approach in the 3D multi-physical sketcher in order to validate the integration of the proposed topological approach in the SAMOS architecture evaluation process.

\section{Conclusions}

This paper focuses on the EMI disturbances evaluation during the conceptual design phase of mechatronic systems. Based on the MBSE SAMOS framework for the 3D architecture evaluation under multi-physical constraints, the proposed approach in this paper focuses on the qualitative and quantitative electromagnetic interferences (EMI) assessment, notably in the case of alien crosstalk of an Electrical Vehicle Power Train. The qualitative evaluation consists of identifying the existence of potential EMI between interacting E/E components. For this purpose, we have developed a topological approach based on the physical and electrical scheme, to automatically detect potential inductive/capacitive crosstalk through an exhaustive loops identification process. Then, the evaluation of these EMI disturbances has been quantitatively performed, based on two approaches as examples: an analytical approach including mathematical rules and physical laws explicitly taking into account the geometry and relative position of the components, and 
a Kron's approach solving a physical problem via a graph by geometrizing the problem and then generating the system of equations associated with this graph. Even though both approaches present the same results regarding the alien crosstalk occurring in an electrical vehicle powertrain, it has been underlined that the analytical approach holds the significant advantage of being able to share geometrical parameters in accordance with the SAMOS collaborative process, in order on the one hand, to compare them with the requirements of the system architect, and on the other hand, to share them with the 3D architect in charge of designing the $3 \mathrm{D}$ architecture in this environment.

\section{References}

[1] A. Kossiakoff, W.N. Sweet, S.J. Seymour, S.M. Biemer, Systems engineering principles and practice, John Wiley \& Sons, 2011

[2] R. Plateaux, O. Penas, J.-Y. Choley, F.M. M'henni, A. Rivière, Integrated design methodology of a mechatronic system, Mécanique \& Industries 11, 401-406 (2010)

[3] G. Hamza, M. Hammadi, M. Barkallah, J.-Y. Choley, A. Riviere, J. Louati, M. Haddar, Conceptual design methodology for the preliminary study of a mechatronic system: application to wind turbine system, Mechanics \& Industry 18, 413 (2017)

[4] J.F. Mologni, M. Bonadiman, A.C. Guimaraes, L. Alvarenga, A. Colin, J.O.S. Paulino, Accelerating the vehicle development process by employing EMI and EMC numerical analysis assisted by high performance computing, SAE International, Warrendale, PA, 2010

[5] Y. Zhang, X. Zhang, Y. Ding, L. Jiang, Research on electromagnetic compatibility of new energy vehicles, DEStech Transactions on Engineering and Technology Research 0 (2017). https://doi.org/10.12783/dtetr/icv mee2017/14638

[6] D.N.J. Carter, The past, present and future challenges of aircraft EMC, IEEE Electromagnetic Compatibility Magazine 1, 4 (2012)

[7] S. Ranganathan, D.G. Beetner, R. Wiese, T.H. Hubing, An expert system architecture to detect system-level automotive EMC problems, in: IEEE, 2002, pp. 976-981

[8] T. Rybak, M. Steffka, Automotive electromagnetic compatibility (EMC), Springer Science \& Business Media, 2004

[9] H. Zhao, G. Li, N. Wang, S. Zheng, L. Yu, Y. Chen, Study of EMC problems with vehicles, in: Information computing and applications, Springer, Berlin, Heidelberg, 2013, pp. 159-168

[10] B. Vrignon, P. Caunegre, J. Shepherd, J. Wu, Automatic verification of EMC immunity by simulation, in: 2013 9th International Workshop on Electromagnetic Compatibility of Integrated Circuits (EMC Compo), 2013, pp. 202-207

[11] K. Burnham, Automotive system integration for EMC, in: 2017 IEEE International Symposium on Electromagnetic Compatibility Signal/Power Integrity (EMCSI), 2017, pp. $1-20$

[12] L. Wang, W. Shen, H. Xie, J. Neelamkavil, A. Pardasani, Collaborative conceptual designðstate of the art and future trends, 16 (2002)
[13] N. Perry, M. Mauchand, A. Bernard, Costs models in design and manufacturing of sand casting products, in: A. Bramley, D. Brissaud, D. Coutellier, C. McMahon (Eds.), Advances in Integrated Design and Manufacturing in Mechanical Engineering Springer-Verlag, Berlin/Heidelberg, 2005, pp. 69-80

[14] J.A. Crowder, J.N. Carbone, R. Demijohn, Multidisciplinary systems engineering: architecting the design process, Springer, 2015

[15] M. Kharrat, O. Penas, R. Plateaux, H. Trabelsi, J.Y. Choley, J. Louati, M. Haddar, Towards a 3D conceptual architecture framework, based on multi-physical constraints, in: 2017 IEEE International Systems Engineering Symposium (ISSE), 2017, pp. 1-8

[16] C. Capasso, M. Hammadi, S. Patalano, R. Renaud, O. Veneri, A multi-domain modelling and verification procedure within MBSE approach to design propulsion systems for road electric vehicles, Mechanics \& Industry 18, 107 (2017)

[17] R. Barbedienne, Y.B. Messaoud, J.Y. Choley, O. Penas, A. Ouslimani, A. Rivière, SAMOS for spatial architecture based on multi-physics and organisation of systems in conceptual design, in: 2015 IEEE International Symposium on Systems Engineering (ISSE), 2015, pp. 135-141

[18] R. Barbedienne, O. Penas, J.Y. Choley, L. Gasser, TheReSE: SysML extension for thermal modeling, in: 2015 Annual IEEE Systems Conference (SysCon) Proceedings, Vancouver, BC, 2015, pp. 301-308

[19] R. Plateaux, Continuité et cohérence d'une modélisation des systèmes mécatroniques basée(s) sur une structure topologique, $\mathrm{PhD}$ Thesis, Ecole Centrale Paris, 2011

[20] M.M. Chaabane, Modélisation géométrique et mécanique pour les systèmes mécatroniques, $\mathrm{PhD}$ Thesis, École Centrale Paris, 2014

[21] N. Abdeljabbar Kharrat, R. Plateaux, M. Miladi Chaabane, J.-Y. Choley, C. Karra, M. Haddar, Integration of topological modification within the modeling of multi-physics systems: application to a Pogo-stick, Comptes Rendus Mécanique 346, 351-365 (2018)

[22] O. Penas, R. Plateaux, J.Y. Choley, A. Rivière, The different complexity levels in mechatronic design process, in: 3rd International Conference on Software, Knowledge, Information Management and Applications SKIMA, Fès, Morocco, 2009

[23] R. Plateaux, J.Y. Choley, O. Penas, A. Riviere, Towards an integrated mechatronic design process, in: 2009 IEEE International Conference on Mechatronics, 2009, pp. 1-6

[24] M.M. Chaabane, R. Plateaux, J.-Y. Choley, C. Karra, A. Rivière, M. Haddar, Topological approach to solve frame structures using topological collections and transformations, Comptes Rendus Mécanique 342, 466-477 (2014)

[25] N. Abdeljabbar Kharrat, R. Plateaux, M. Miladi Chaabane, J.-Y. Choley, C. Karra, M. Haddar, Topological modeling of 2D piezoelectric truss structure using the MGS language, in: M. Haddar, F. Chaari, A. Benamara, M. Chouchane, C. Karra, N. Aifaoui (Eds.), Design and Modeling of Mechanical Systems-III, Springer International Publishing, Cham 2018, pp

[26] C.R. Paul, R.T. Abraham, Coupling of electromagnetic fields to transmission lines, in: 1981 IEEE International Symposium on Electromagnetic Compatibility, 1981, pp. 1-7 
[27] R.F. Harrington, Field computation by moment methods, Wiley-IEEE Press, 1993

[28] K.S. Kunz, R.J. Luebbers, The finite difference time domain method for electromagnetics, CRC press, 1993

[29] C.R. Paul, Analysis of multiconductor transmission lines, John Wiley \& Sons, 2008

[30] O. Maurice, La compatibilité électromagnétique des systèmes complexes, Lavoisier, Cachan, 2007

[31] O. Maurice, Quelques exemples d'analyses théoriques en compatibilité électromagnétique, Bookelis, 2018

[32] P. Kirawanich, N. Kranthi, N.E. Islam, S.J. Yakura, Electromagnetic topology-based analysis of coupling through small aperture on cables of communication systems, Electromagnetics 25, 589-602 (2005)

[33] J. Parmantier, Numerical coupling models for complex systems and results, IEEE Transactions on Electromagnetic Compatibility 46, 359-367 (2004)

[34] P. Besnier, Electromagnetic topology: an additional interaction sequence diagram for transmission line network analysis, IEEE Transactions on Electromagnetic Compatibility 48, 685-692 (2006)

[35] J.-P. Parmantier, I. Junqua, EM topology: from theory to application, in: Ultra-Wideband, Short-Pulse Electromagnetics 7, Springer, 2007, pp. 3-12

[36] C.E. Baum, Electromagnetic topology for the analysis and design of complex electromagnetic systems, in: Fast Electrical and Optical Measurements, Springer, 1986, pp. 467-547

[37] C.E. Baum, How to think about EMP interaction, in: Proceedings of the1974 Spring FULMEN Meeting, 1974

[38] F.M. Tesche, Topological concepts for internal EMP interaction, IEEE Transactions on Electromagnetic Compatibility, 60-64 (1978)

[39] E. Genender, H. Garbe, F. Sabath, Probabilistic risk analysis technique of intentional electromagnetic interference at system level, IEEE Transactions on Electromagnetic Compatibility 56, 200-207 (2014)

[40] O. Maurice, Adaptation of Kron's tensorial analysis of network for the EMC design and analysis of systems, (2016). https://hal.archives-ouvertes.fr/hal-01382221 (accessed May 9, 2018)

[41] R. Barbedienne, O. Penas, J.Y. Choley, A. Rivière, A. Warniez, F.D. Monica, Introduction of geometrical contraints modeling in SysML for mechatronic design, in: 2014 10th France-Japan/8th Europe-Asia Congress on Mecatronics (MECATRONICS2014- Tokyo), Tokyo, Japan, 2014, pp. 145-150
[42] M. Mardiguian, Manuel pratique de compatibilité électromagnétique: prédictions et solutions aux perturbations électromagnétiques, Lavoisier, 2003

[43] J.Y. Lim, K.Y. See, E.K. Chua, Impact of circuit impedance on signal crosstalk, in: 2015. https:/ /www.researchgate.net/ publication/280495863_Impact_of_Circuit_Impedan ce on Signal Crosstalk

[44] M. Kharrat, $\bar{N}$. Abdeljabbar, O. Penas, R. Plateaux, J. Louati, M. Miladi Chaaben, J.-Y. Choley, M. Haddar, EMC risk assessment process through a topological analysis, 12th France-Japan/10th Europe-Asia Congress on Mecatronics (Mie, Japan MECATRONICS 2018), Submitted. (2018)

[45] M. Reuter, S. Tenbohlen, W. Köhler, Influence of a traction battery's input impedance on conducted emissions of an automotive HV inverter, in: 2013 International Symposium on Electromagnetic Compatibility, 2013, pp. 229-234

[46] B. Willmann, T. Rinkleff, M. Obholz, R. Vick, Automotive industry's EMC requirements for voltage ripple in the high-voltage system of electrical vehicles, in: IEEE, 2015, pp. $673-678$

[47] M. Kharrat, O. Penas, R. Plateaux, J.-Y. Choley, H. Trabelsi, J. Louati, M. Haddar, Integration of electromagnetic constraints as of the conceptual design through an MBSE approach, IEEE Systems Journal, 1-12 (2020)

[48] O. Maurice, Theoretical application of the tensorial analysis of network for EMC at the system level (2007)

[49] G. Kron, Tensors for circuits, Dover Publications, 1959. http://archive.org/details/TensorsForCircuits (accessed December 24, 2018)

[50] O. Maurice, Compatibilité électromagnétique - Notions fondamentales, Techniques de l'ingénieur. E1302 v2 (2016)

[51] G. Oliva, R. Setola, L. Glielmo, C.N. Hadjicostis, Distributed cycle detection and removal, IEEE Transactions on Control of Network Systems 5, 194-204 (2018)

[52] E.W. Dijkstra, C.S. Scholten, Termination detection for diffusing computations, Information Processing Letters 11, $1-4(1980)$

[53] M. Safar, K. Alenzi, S. Albehairy, Counting cycles in an undirected graph using DFS-XOR algorithm, in: 2009 First International Conference on Networked Digital Technologies, IEEE, 2009, pp. 132-139

[54] C. Cassiolato, Inductive coupling and how to minimize their effects in industrial installations | SMAR - Industrial Automation, SMAR (2011). http://www.smar.com/en/ technical-article/inductive-coupling-and-how-to-minimizetheir-effects-in-industrial-installations (accessed December $6,2018)$

Cite this article as: M. Kharrat, O. Penas, N. Abdeljabbar Kharrat, R. Plateaux, J.-Y. Choley, J. Louati, H. Trabelsi, M. Haddar, Topological approach for assessment of electromagnetic interferences to support mechatronic conceptual design, Mechanics \& Industry 21, 609 (2020) 